\title{
STOCHASTIC AND VARIATIONAL APPROACH TO THE LAX-FRIEDRICHS SCHEME
}

\author{
KOHEI SOGA
}

\begin{abstract}
In this paper we present a stochastic and variational aspect of the Lax-Friedrichs scheme applied to hyperbolic scalar conservation laws. This is a finite difference version of Fleming's results ('69) that the vanishing viscosity method is characterized by stochastic processes and calculus of variations. We convert the difference equation into that of the Hamilton-Jacobi type and introduce corresponding calculus of variations with random walks. The stability of the scheme is obtained through the calculus of variations. The convergence of approximation is derived from the law of large numbers in hyperbolic scaling limit of random walks. The main advantages due to our approach are the following: Our framework is basically a.e. pointwise convergence with characterization of "a.e.", which yields uniform convergence except "small" neighborhoods of shocks; The stability and convergence proofs are verified for arbitrarily large time interval, which are hard to obtain in the case of flux functions of general types depending on both space and time; the approximation of characteristic curves is available as well as that of PDE-solutions, which is particularly important for applications of the Lax-Friedrichs scheme to the weak KAM theory.
\end{abstract}

\section{INTRODUCTION}

The Lax-Friedrichs scheme is one of the oldest, simplest and most universal techniques for computing PDEs. There is a huge body of literature on the stability and convergence of the Lax-Friedrichs scheme based on the $L^{1}$-framework, particularly for shock waves. In this paper we investigate the Lax-Friedrichs scheme in terms of scaling limit of random walks and calculus of variations, and present several useful new results relating the scheme to principles of probability theories and calculus of variations. We refer to only the Lax-Friedrichs scheme applied to hyperbolic scalar conservation laws, but the same results for other schemes with numerical viscosity (e.g., up/down wind schemes) are available. Moreover, our results imply that a wide class of finite difference methods applied to evolution equations is likely to possess stochastic or variational structures. It is a meaningful effort to investigate the Lax-Friedrichs scheme or other schemes applied to PDEs of various types by a similar approach to ours.

Before going into detail, let us place our new framework in the huge body of literature of analysis of PDEs. The various methods of PDEs can be roughly classified into the two groups: the PDE approach that is based on theories of functional

Received by the editor March 28, 2012 and, in revised form, April 23, 2013, and June 6, 2013. 2010 Mathematics Subject Classification. Primary 65M06, 35L65, 49L25, 60G50.

Key words and phrases. Lax-Friedrichs scheme, scalar conservation law, Hamilton-Jacobi equation, calculus of variations, random walk, law of large numbers.

The author was supported by Grant-in-Aid for JSPS Fellows (20-6856). 
analysis and the dynamical approach that is based on theories of dynamical systems and stochastic processes. The dynamical approach is not merely a recast of the PDE approach. It often provides new viewpoints and detailed information through techniques and notions of calculus of variations, probability theories, etc., which are invisible and unavailable in the PDE approach. We point out several examples of great successes due to the dynamical approach: 9, 10 for connections between parabolic PDEs and optimal control problems; 7], 8], 6] for connections among first order Hamilton-Jacobi equations, hyperbolic conservation laws and Hamiltonian/Lagrangian dynamics (the weak KAM theory); 2, [1], 11] for viscosity and discount approximation methods in the weak KAM theory. To the best of my knowledge, there is no dynamical approach to finite difference methods in the existing literature. The purpose of this paper is to make a fundamental investigation of the possibility of introducing the dynamical approach into finite difference methods and to show some development in stability and convergence arguments due to the dynamical approach.

We consider initial value problems of the inviscid hyperbolic scalar conservation law

$$
\left\{\begin{array}{l}
u_{t}+H(x, t, c+u)_{x}=0 \text { in } \mathbb{T} \times(0, T] \\
u(x, 0)=u^{0}(x) \in L^{\infty}(\mathbb{T}) \text { on } \mathbb{T}, \quad \int_{\mathbb{T}} u^{0}(x) d x=0
\end{array}\right.
$$

where $c$ is a parameter varying within an interval $\left[c_{0}, c_{1}\right]$ and $\mathbb{T}:=\mathbb{R} / \mathbb{Z}$ is the standard torus. The assumptions for the flux function $H$ are the following (A1)-(A4):

$$
\begin{aligned}
& \text { (A1) } H(x, t, p): \mathbb{T}^{2} \times \mathbb{R} \rightarrow \mathbb{R}, C^{2}, \\
& \text { (A2) } H_{p p}>0, \\
& \text { (A3) } \lim _{|p| \rightarrow+\infty} \frac{H(x, t, p)}{|p|}=+\infty .
\end{aligned}
$$

By (A1)-(A3), we have the Legendre transform $L(x, t, \xi)$ of $H(x, t, \cdot)$, which is now given by

$$
L(x, t, \xi)=\sup _{p \in \mathbb{R}}\{\xi p-H(x, t, p)\}
$$

and satisfies

$$
\begin{aligned}
& \text { (A1)' } L(x, t, \xi): \mathbb{T}^{2} \times \mathbb{R} \rightarrow \mathbb{R}, C^{2}, \\
& \text { (A2)' } L_{\xi \xi}>0, \\
& \text { (A3)' } \lim _{|\xi| \rightarrow+\infty} \frac{L(x, t, \xi)}{|\xi|}=+\infty .
\end{aligned}
$$

The last assumption is:

(A4) There exists $\alpha>0$ such that $\left|L_{x}\right| \leq \alpha(|L|+1)$.

Throughout this paper, $\mathbb{T}$-dependency is identified with $\mathbb{R}$-dependency with $\mathbb{Z}$ periodicity and $\mathbb{T}$ with $[0,1)$. (A1) and (A2) are standard in the theories of conservation laws. (A3) is necessary, when we introduce the variational approach, stated below, to our problems. (A4) is used for derivation of boundedness of minimizers of the variational problems. The problems (1.1) appear not only in continuum mechanics but also in Hamiltonian and Lagrangian dynamics generated by $H$ and $L$ [7, [12, 6], 17]. In the latter case the periodic setting is standard. We remark that the whole space setting is also available with additional assumptions for $H$ required by variational techniques. 
It is sometimes very convenient to introduce initial value problems of HamiltonJacobi equations which are equivalent to (1.1)

$$
\left\{\begin{array}{l}
v_{t}+H\left(x, t, c+v_{x}\right)=h(c) \text { in } \mathbb{T} \times(0, T], \\
v(x, 0)=v^{0}(x) \in \operatorname{Lip}(\mathbb{T}) \text { on } \mathbb{T},
\end{array}\right.
$$

where $h:\left[c_{0}, c_{1}\right] \rightarrow \mathbb{R}$ is a given function. We consider (1.1) and (1.2) in the class of generalized solutions called entropy solutions and viscosity solutions respectively. Such solutions exist in $C^{0}\left((0, T] ; L^{1}(\mathbb{T}) \cap L^{\infty}(\mathbb{T})\right)$ and $\operatorname{Lip}(\mathbb{T} \times(0, T])$. If $u^{0}=v_{x}^{0}$, then the entropy solution $u$ of (1.1) and the viscosity solution $v$ of (1.2) satisfy $u=v_{x}$ ( $u$ is considered as a representative element). From now on we always assume that $u^{0}=v_{x}^{0}$.

The dynamical approach provides one of the central achievements in the analysis of (1.1) and (1.2), relating them to deterministic calculus of variations in Lagrangian dynamics: The value of $v$ at each point $(x, t) \in \mathbb{T} \times(0, T]$ is given by

(1.3) $v(x, t)=\inf _{\gamma \in A C, \gamma(t)=x}\left\{\int_{0}^{t} L^{c}\left(\gamma(s), s, \gamma^{\prime}(s)\right) d s+v^{0}(\gamma(0))\right\}+h(c) t$,

where $A C$ is the family of absolutely continuous curves $\gamma:[0, t] \rightarrow \mathbb{R}$ and

$$
L^{c}(x, t, \xi):=L(x, t, \xi)-c \xi
$$

is the Legendre transform of $H(x, t, c+\cdot)$ (see e.g. 3]). We can find a minimizing curve $\gamma^{*}$ of (1.3), which is a backward characteristic curve of $u, v$ and a $C^{2}$-solution of the Euler-Lagrange equation associated with the Lagrangian $L^{c}$. If the point $(x, t)$ is a regular point of $v$ (i.e., $v_{x}(x, t)$ exists), then the value $u(x, t)=v_{x}(x, t)$ is given by

$$
u(x, t)=\int_{0}^{t} L_{x}^{c}\left(\gamma^{*}(s), s, \gamma^{* \prime}(s)\right) d s+u^{0}\left(\gamma^{*}(0)\right),
$$

where $u^{0}$ is supposed to be rarefaction-free, or equivalently $v^{0}$ is semiconcave. (Otherwise, $u^{0}\left(\gamma^{*}(0)\right)$ needs to be replaced with $L_{\xi}^{c}\left(\gamma^{*}(0), 0, \gamma^{* \prime}(0)\right)$; see Lemma 3.2.) We remark that, since $v$ is Lipschitz, almost every point is regular and (1.4) is valid for almost every point.

The representation formula (1.3) is a strong tool not only in the analysis of (1.1) and (1.2) but also in many applications of the equations to other fields. It should be noted that analysis of (1.1) and (1.2) based on (1.3) and (1.4) is a starting point of the dynamical approach to approximation theories of (1.1) and (1.2) in terms of the vanishing viscosity method and finite difference methods. The first case is announced by Fleming [9] and the latter one is the theme of this paper.

First we recall the results of Fleming. Let us consider initial value problems of

$$
\begin{aligned}
& u_{t}^{\nu}+H\left(x, t, c+u^{\nu}\right)_{x}=\nu u_{x x}^{\nu}, \\
& v_{t}^{\nu}+H\left(x, t, c+v_{x}^{\nu}\right)=h(c)+\nu v_{x x}^{\nu} \quad(\nu>0),
\end{aligned}
$$

with the same setting as (1.1) and (1.2). The solutions $u^{\nu}$ and $v^{\nu}$ are also related to calculus of variations which are not deterministic but stochastic: The value of $v^{\nu}$ at each point $(x, t) \in \mathbb{T} \times(0, T]$ is given by

$$
\begin{aligned}
& v^{\nu}(x, t)=\inf _{\xi^{\nu} \in C^{1}\left(\mathbb{T}^{2}\right)} E\left[\int_{0}^{t} L^{c}\left(\gamma^{\nu}(s), s, \xi^{\nu}\left(\gamma^{\nu}(s), s\right)\right) d s+v^{0}\left(\gamma^{\nu}(0)\right)\right] \\
& +h(c) t \text {, }
\end{aligned}
$$


where $E$ stands for the expectation with respect to the Wiener measure and $\gamma^{\nu}$ is a solution of the stochastic ODE

$$
d \gamma^{\nu}(s)=\xi^{\nu}\left(\gamma^{\nu}(s), s\right) d s-\sqrt{2 \nu} d B(t-s), \quad \gamma^{\nu}(t)=x .
$$

Here $B$ is the standard Brownian motion. There exists the unique minimizing vector field $\xi^{\nu *}$ of (1.7). The value $u^{\nu}(x, t)$ is given by

$$
u^{\nu}(x, t)=E\left[\int_{0}^{t} L_{x}^{c}\left(\gamma^{\nu *}(s), s, \xi^{\nu *}\left(\gamma^{\nu *}(s), s\right)\right) d s+u^{0}\left(\gamma^{\nu *}(0)\right)\right],
$$

where $\gamma^{\nu *}$ is a solution of (1.8) with $\xi^{\nu}=\xi^{\nu *}$. It is proved from a stochastic and variational point of view that, for $\nu \rightarrow 0+, v^{\nu}$ converges uniformly to $v$ with the error $O(\sqrt{\nu})$ and $u^{\nu}$ converges pointwise to $u=v_{x}$ except for points of discontinuity of $u$. In particular, $u^{\nu}$ converges uniformly to $u$ without an arbitrarily small neighborhood of shocks. The proof indicates how the stochastic variational formulas (1.7) and (1.9) tend to the deterministic ones, (1.3) and (1.4). Asymptotics of (1.8) for $\nu \rightarrow 0$ plays a central role. Fleming's approach yields much information and concrete pictures of the vanishing viscosity method. In particular, we can see how the parabolicity disappears to be hyperbolic.

Now we observe the basic idea of the dynamical approach to finite difference methods with the Lax-Friedrichs scheme. We discretize the equation of (1.1) by the Lax-Friedrichs scheme:

$$
\text { (1.10) } \frac{u_{m+1}^{k+1}-\frac{\left(u_{m}^{k}+u_{m+2}^{k}\right)}{2}}{\Delta t}+\frac{H\left(x_{m+2}, t_{k}, c+u_{m+2}^{k}\right)-H\left(x_{m}, t_{k}, c+u_{m}^{k}\right)}{2 \Delta x}=0 \text {. }
$$

We will see in the next section that we can find a difference equation which approximates the equation of (1.2) and is equivalent to (1.10) in the sense that $u_{m}^{k}=\left(v_{m+1}^{k}-v_{m-1}^{k}\right) / 2 \Delta x$ :

$$
\frac{v_{m}^{k+1}-\frac{\left(v_{m-1}^{k}+v_{m+1}^{k}\right)}{2}}{\Delta t}+H\left(x_{m}, t_{k}, c+\frac{v_{m+1}^{k}-v_{m-1}^{k}}{2 \Delta x}\right)=h(c) .
$$

We present stochastic calculus of variations associated with (1.11) that we minimize the expectation of a discrete action functional among space-time inhomogeneous random walks in $\Delta x \mathbb{Z} \times \Delta t \mathbb{Z}$. This yields representation formulas of $v_{m+1}^{k}$ and $u_{m}^{k}$ similar to (1.7) and (1.9). The probability measures of such random walks are no longer related to the Wiener measure. This is the main difficulty of our arguments: We need to study the asymptotics for $\Delta x, \Delta t \rightarrow 0$ of the random walks generated by arbitrary transition probabilities depending on space and time, under hyperbolic scaling $0<\lambda_{0} \leq \Delta t / \Delta x \leq \lambda_{1}$. We will see that the continuous limit of minimizing random walks is deterministic. In other words, we obtain the law of large numbers, where the minimizing random walks converge to the minimizing curves for $u, v$. This proves convergence of the Lax-Friedrichs scheme and a boundedness property of minimizing random walks proves stability of the scheme. It is interesting to note that, under diffusive scaling $\Delta x^{2} / \Delta t=2 \nu>0$, the continuous limit of a certain class of random walks is the Brownian motion or diffusion processes, and the solutions of (1.10) and (1.11) converge to these of (1.5) and (1.6). It is expected that the convergence of the scheme can be characterized by the central limit theorem, though we do not go into the diffusive case in this paper.

Our approach also yields much information and concrete pictures of the finite difference method with the Lax-Friedrichs scheme. In particular, we can see how the 
"parabolicity" due to numerical viscosity disappears to be hyperbolic in terms of the law of large numbers. Here we point out several advantages due to our approach:

(1) Stability of the Lax-Friedrichs scheme and therefore convergence of the scheme, up to arbitrary $T>0$, is derived from variational techniques.

(2) A.e. pointwise convergence of $u_{m}^{k}$ to $u=v_{x}$ is proved with characterization of "a.e.". In particular, this yields the uniform convergence except arbitrarily small neighborhoods of shocks with characterization of "shock".

(3) The uniform convergence of $v_{m+1}^{k}$ to $v$ with an error $O(\sqrt{\Delta x})$ is proved from the stochastic viewpoint.

(4) The approximation of (backward) characteristic curves of (1.1) and (1.2) and its convergence are verified.

The existing literature on the Lax-Friedrichs scheme (also with other schemes) is based on the PDE approach, where we have the well-organized $L^{1}$-framework with compactness of functions of bounded variation or compensated compactness. If we assume strict convexity of flux functions, we can use compactness of functions of bounded variation and can easily prove $L^{1}$ and a.e. pointwise convergence. We remark that, in the PDE approach, an entropy solution is an $\left(L^{1} \cap L^{\infty}\right)$-valued function and there is no reason to specify a representative element and to characterize "a.e.", while, in the dynamical approach, an entropy solution can be taken as the derivative of the corresponding viscosity solutions defined for a.e. points. Here "a.e." and "shocks" of the entropy solution mean the set of points of singularity of the viscosity solutions. In order to obtain convergence of schemes based on the $L^{1}$-framework, we need to confirm mesh-size independent boundedness of difference solutions (stability of schemes). This part gets much harder in the case of $(x, t)$-dependent flux functions. We briefly review the PDE approach to the finite difference methods applied to conservation laws.

In the case of flux functions which are independent of $x$ and $t$, many details are known. Crandall and Majda [5] prove stability and $L^{1}$-convergence properties of monotone difference approximations in a rather general setting, where a flux function $H(p)$ is not necessarily convex. Tadmor [21] shows the one-sided Lipschitz condition $\left(u_{m+2}^{k}-u_{m}^{k}\right) / 2 \Delta x \leq \frac{a}{k \Delta t}$, which guarantees time-global stability. Şabac [16] proves that the optimal $L^{1}$-convergence rate of $u_{m}^{k} \rightarrow u$ is $O(\sqrt{\Delta x})$.

In the case of flux functions depending on both $x$ and $t$, additional assumptions are required so far: Oleinik [15] extensively investigates the Lax-Freidrichs scheme in this case, where stability and $L^{1}$-convergence are proved with a restricted $T>$ 0 . This restriction is undesirable, because, if we expect $\left|u_{m}^{k}\right| \leq M$ within $[0, T]$, admissible $T$ must be less than $\int_{\left\|u^{0}\right\|_{L} \infty}^{M} \frac{1}{V(r)+b} d r$ with a certain constant $b>0$ and a monotone increasing $C^{1}$-function $V$ such that $\sup _{x, t \in \mathbb{T},|p| \leq r}\left|H_{x}(x, t, p)\right| \leq V(r)$ $\left(V(r)=a r^{2}\right.$ is often the case, due to $H_{p p}>0$, with $\left.\int_{\left\|u^{0}\right\|_{L^{\infty}}}^{\infty} \frac{1}{V(r)+b} d r<\infty\right)$. For such a $T$, a priori boundedness of difference solutions is proved within $[0, T]$. Nishida and Soga [14] show the time-global stability of the Lax-Freidrichs scheme as well as the long time behavior that any difference solution converges exponentially to a periodic state as $k \rightarrow \infty$ yielding $\mathbb{Z}^{2}$-periodic entropy solutions. This argument is a generalization of the results of Oleinik [15] and Tadmor [21. However, they still assume that the flux function is of the form $H(x, t, p)=g_{1}(p)+g_{2}(x, t)$ in order to prove a priori boundedness of difference solutions. Karlsen and Towers 13. prove convergence of the Lax-Friedrichs scheme for flux functions of the form 
$H(x, t, p)=f(k(x, t), p)$ allowing nonconvex dependence with respect to $p$ and discontinuous functions $k(x, t)$. They use a strong condition for $f$ (conditions (2.1) and (2.5) in [13]) that is almost equivalent to assuming boundedness of difference solutions and therefore stability of the scheme in advance. Our current setting (A1)-(A4) is completely out of this condition. It seems extremely hard to prove stability of the Lax-Friedrichs scheme for arbitrary $T>0$ with $H(x, t, p)$ satisfying (A1)-(A4) in the PDE approach.

This difficulty will be removed in the dynamical approach with the aid of calculus of variation, which is one of the important consequences of our new framework. Once we establish a priori boundedness, a convergence proof is rather straightforward in the PDE approach, even under (A1)-(A4). However, we will give a convergence proof based on the law of large numbers in random walks, without any compactness arguments. As a result, we obtain not only a.e. pointwise convergence to exact entropy solutions with the characterization of "a.e." but also convergence of random walks to their characteristic curves, which is another important outcome of the dynamical approach. We discuss this point in terms of application to the weak KAM theory.

There are also many works on finite difference approximation to viscosity solutions of Hamilton-Jacobi equations in the PDE approach. Many of them are done independently of entropy solutions. We remark that, even in the case of (1.1) and (1.2), convergence results for viscosity solutions do not necessarily imply these for entropy solutions. We point out Crandall and Lions 4 and Souganidis 20, where convergence results for viscosity solutions with general schemes are established as well as error estimates of $O(\sqrt{\Delta x})$. Our results provide interpretation of the order $\sqrt{\Delta x}$ from the stochastic viewpoint.

The dynamical approach to finite difference methods also leads to successful applications to the weak KAM theory, which would not be expected in the PDE approach. The weak KAM theory clarifies the important connection among entropy solutions, viscosity solutions and the Hamiltonian/Lagrangian dynamics generated by $H(x, t, p)$ through the dynamical approach (Lax-Oleinik type operators) to Hamilton-Jacobi equations. Now let us consider approximation methods for the weak KAM theory. Since compatibility with the exact weak KAM framework is required, approximation methods of the weak KAM theory should be kinds of the dynamical approach. Furthermore, since the central objects of the weak KAM theory are entropy solutions, viscosity solutions and their characteristic curves, such approximation methods should provide all of them at the same time. In particular, approximation of characteristic curves is very important. The vanishing viscosity method meets the requirements through Fleming's results. Bessi 2 and Anantharaman et al. [1] exploit Fleming's approach to develop smooth approximation in the weak KAM theory. The discount approximation method in terms of dynamical approach is also successfully applied to the weak KAM theory 11. Nishida and Soga [14] develop difference approximation in the weak KAM theory by the LaxFriedrichs scheme with the PDE approach (not the dynamical approach). They perform a rather nice numerical computation of the Aubry-Mather sets (one of the central objects in the weak KAM theory), but mathematical justification is not done due to the lack of the above requirement. Our new framework possessing (1)-(4) meets the above requirement. In fact, the author recently announced 
an extensive application of the results of the present paper to the weak KAM theory [19. We remark that the Aubry-Mather sets sometimes have zero-measure and structures similar to Cantor sets. Therefore we have to be careful about the treatment of "a.e." in the weak KAM theory. As mentioned above, our new framework characterizes "a.e.", which would be convenient there.

\section{RESUlts}

Here are our assumptions required by the following results:

Assumption. Suppose (A1)-(A4). Let $T>0$ be arbitrarily fixed. The parameter $c$ varies within $\left[c_{0}, c_{1}\right]$. Initial data are bounded: $\left\|u^{0}\right\|_{L^{\infty}}=\left\|v_{x}^{0}\right\|_{L^{\infty}} \leq r$, $\|$ $v^{0} \|_{C^{0}(\mathbb{T})}:=\sup _{x \in \mathbb{T}}\left|v^{0}(x)\right| \leq r$. Here $c_{0}, c_{1}$ and $r>0$ are arbitrarily given numbers.

Note that $\left\|v^{0}\right\|_{C^{0}(\mathbb{T})} \leq r$ is not essential, since we can subtract any number from solutions of Hamilton-Jacobi equations.

2.1. Equivalent schemes for conservation laws and Hamilton-Jacobi equations. Let $N, K$ be natural numbers. The mesh size $\Delta=(\Delta x, \Delta t)$ is defined by $\Delta x:=(2 N)^{-1}$ and $\Delta t:=(2 K)^{-1}$. Set $\lambda:=\Delta t / \Delta x, x_{m}:=m \Delta x$ for $m \in \mathbb{Z}$ and $t_{k}:=k \Delta t$ for $k=0,1,2, \cdots$. For $x \in \mathbb{R}$ and $t>0$, the notation $m(x), k(t)$ denote the integers $m, k$ for which $x \in\left[x_{m}, x_{m}+2 \Delta x\right), t \in\left[t_{k}, t_{k}+\Delta t\right)$. Let $(\Delta x \mathbb{Z}) \times\left(\Delta t \mathbb{Z}_{\geq 0}\right)$ be the set of all $\left(x_{m}, t_{k}\right)$ and let

$$
\mathcal{G}_{\text {even }} \subset(\Delta x \mathbb{Z}) \times\left(\Delta t \mathbb{Z}_{\geq 0}\right), \quad \mathcal{G}_{\text {odd }} \subset(\Delta x \mathbb{Z}) \times\left(\Delta t \mathbb{Z}_{\geq 0}\right)
$$

be the set of all $\left(x_{m}, t_{k}\right)$ with $k=0,1,2, \cdots$ and $m \in \mathbb{Z}$ with $m+k=$ even, odd. We call $\mathcal{G}_{\text {even }}, \mathcal{G}_{\text {odd }}$ the even grid, odd grid. We consider the discretization of (1.1) by the Lax-Freidrichs scheme in $\mathcal{G}_{\text {even }}$ :

$$
\left\{\begin{array}{l}
\frac{u_{m+1}^{k+1}-\frac{\left(u_{m}^{k}+u_{m+2}^{k}\right)}{2}}{\Delta t}+\frac{H\left(x_{m+2}, t_{k}, c+u_{m+2}^{k}\right)-H\left(x_{m}, t_{k}, c+u_{m}^{k}\right)}{2 \Delta x}=0, \\
u_{m}^{0}=u_{\Delta}^{0}\left(x_{m}\right), \quad u_{m \pm 2 N}^{k}=u_{m}^{k}
\end{array}\right.
$$

where for $m=$ even,

$$
u_{\Delta}^{0}(x):=\frac{1}{2 \Delta x} \int_{x_{m}-\Delta x}^{x_{m}+\Delta x} u^{0}(y) d y \text { for } x \in\left[x_{m}-\Delta x, x_{m}+\Delta x\right) .
$$

Note that $\sum_{\{m \mid 0 \leq m<2 N, m+k=e v e n\}} u_{m}^{k} \cdot 2 \Delta x$ is conservative with respect to $k$ and is zero for $u^{0}$ with the average zero. Now we consider a discrete version of (1.2) in $\mathcal{G}_{\text {odd }}$ :

$$
\left\{\begin{array}{l}
\frac{v_{m}^{k+1}-\frac{\left(v_{m-1}^{k}+v_{m+1}^{k}\right)}{\Delta t}}{2}+H\left(x_{m}, t_{k}, c+\frac{v_{m+1}^{k}-v_{m-1}^{k}}{2 \Delta x}\right)=h(c), \\
v_{m+1}^{0}=v_{\Delta}^{0}\left(x_{m+1}\right), \quad v_{m+1 \pm 2 N}^{k}=v_{m+1}^{k},
\end{array}\right.
$$

where $v_{\Delta}^{0}$ is a function which converges to $v^{0}$ uniformly as $\Delta \rightarrow 0$. We introduce the difference operators:

$$
D_{t} w_{m}^{k+1}:=\frac{w_{m}^{k+1}-\frac{w_{m-1}^{k}+w_{m+1}^{k}}{2}}{\Delta t}, \quad D_{x} w_{m+1}^{k}:=\frac{w_{m+1}^{k}-w_{m-1}^{k}}{2 \Delta x} .
$$

With the assumption $u^{0}=v_{x}^{0}$, we define

$$
v_{\Delta}^{0}(x):=v^{0}(-\Delta x)+\int_{-\Delta x}^{x} u_{\Delta}^{0}(y) d y
$$


i.e., $v_{\Delta}^{0}\left(x_{m+1}\right)=v^{0}\left(x_{m+1}\right)$ for $m$ even. Note that $u_{\Delta}^{0} \rightarrow u^{0}$ in $L^{1}$ and $v_{\Delta}^{0} \rightarrow v^{0}$ uniformly with $\left\|v_{\Delta}^{0}-v^{0}\right\|_{C^{0}} \leq\left\|u^{0}\right\|_{L^{\infty}} \cdot 2 \Delta x$, as $\Delta \rightarrow 0$. The two problems (2.1) and (2.3) are equivalent under (2.2) and (2.4):

Proposition 2.1. Let $u_{m}^{k}$ and $v_{m+1}^{k}$ be the solutions of (2.1) and (2.3) with (2.2) and (2.4). Then the one is derived from the other. In particular, we have $D_{x} v_{m+1}^{k}=$ $u_{m}^{k}$.

2.2. Random walks for the Lax-Friedrichs scheme. We introduce space-time inhomogeneous random walks in $\mathcal{G}_{\text {odd }}$, which corresponds to the stochastic ODE (1.8) in the vanishing viscosity method or characteristic curves of (1.1).

For each point $\left(x_{n}, t_{l+1}\right) \in \mathcal{G}_{\text {odd }}$, we consider backward random walks $\gamma$ which start from $x_{n}$ at $t_{l+1}$ and move by $\pm \Delta x$ in each backward time step:

$$
\gamma=\left\{\gamma^{k}\right\}_{k=0,1, \cdots, l+1}, \quad \gamma^{l+1}=x_{n}, \quad \gamma^{k+1}-\gamma^{k}= \pm \Delta x .
$$

More precisely, we set the following for each $\left(x_{n}, t_{l+1}\right) \in \mathcal{G}_{\text {odd }}$ :

$$
\begin{aligned}
& X^{k}:=\left\{x_{m}\left|\left(x_{m}, t_{k}\right) \in \mathcal{G}_{\text {odd }},\right| x_{m}-x_{n} \mid \leq(l+1-k) \Delta x\right\}, \quad k \leq l+1, \\
& G:=\bigcup_{1 \leq k \leq l+1}\left(X^{k} \times\left\{t_{k}\right\}\right) \subset \mathcal{G}_{\text {odd }}, \\
& \xi: G \ni\left(x_{m}, t_{k}\right) \mapsto \xi_{m}^{k} \in\left[-\lambda^{-1}, \lambda^{-1}\right], \quad \lambda=\Delta t / \Delta x, \\
& \overline{\bar{\rho}}: G \ni\left(x_{m}, t_{k}\right) \mapsto \overline{\bar{\rho}}_{m}^{k}:=\frac{1}{2}-\frac{1}{2} \lambda \xi_{m}^{k} \in[0,1], \\
& \bar{\rho}: G \ni\left(x_{m}, t_{k}\right) \mapsto \bar{\rho}_{m}^{k}:=\frac{1}{2}+\frac{1}{2} \lambda \xi_{m}^{k} \in[0,1], \\
& \gamma:\{0,1,2, \cdots, l+1\} \ni k \mapsto \gamma^{k} \in X^{k}, \gamma^{l+1}=x_{n}, \gamma^{k+1}-\gamma^{k}= \pm \Delta x, \\
& \Omega: \text { the family of } \gamma .
\end{aligned}
$$

We regard $\overline{\bar{\rho}}_{m}^{k}$ (respectively $\left.\bar{\rho}_{m}^{k}\right)$ as a transition probability from $\left(x_{m}, t_{k}\right)$ to $\left(x_{m}+\right.$ $\left.\Delta x, t_{k}-\Delta t\right)$ (from $\left(x_{m}, t_{k}\right)$ to $\left(x_{m}-\Delta x, t_{k}-\Delta t\right)$ ). We control the transition of our random walks by $\xi$, which plays a "velocity field"-like role in $G$. We define the density of each path $\gamma \in \Omega$ as

$$
\mu(\gamma):=\prod_{1 \leq k \leq l+1} \rho\left(\gamma^{k}, \gamma^{k-1}\right),
$$

where $\rho\left(\gamma^{k}, \gamma^{k-1}\right)=\bar{\rho}_{m\left(\gamma^{k}\right)}^{k}$ (respectively $\left.\bar{\rho}_{m\left(\gamma^{k}\right)}^{k}\right)$ if $\gamma^{k}-\gamma^{k-1}=-\Delta x(\Delta x)$. The density $\mu(\cdot)=\mu(\cdot ; \xi)$ yields a probability measure of $\Omega$, namely

$$
\operatorname{prob}(A)=\sum_{\gamma \in A} \mu(\gamma ; \xi) \quad \text { for } A \subset \Omega .
$$

The expectation with respect to this probability measure is denoted by $E_{\mu(\cdot ; \xi)}$, namely for a random variable $f: \Omega \rightarrow \mathbb{R}$,

$$
E_{\mu(\cdot ; \xi)}[f(\gamma)]:=\sum_{\gamma \in \Omega} \mu(\gamma ; \xi) f(\gamma)
$$

The above objects depend on the initial point $\left(x_{n}, t_{l+1}\right)$, but we omit adding the index to them for simpler notation. We remark that, since our transition probabilities are space-time inhomogeneous, the well-known law of large numbers and central limit theorem for random walks do not always hold in our case. Soga 18] 
investigated the asymptotics for $\Delta \rightarrow 0$ of the probability measure of $\Omega$ under hyperbolic scaling, which will be important in this work.

\subsection{Stochastic and variational representation of approximate solutions.}

We represent the approximate solutions by a discrete action functional with the random walks and $L^{c}=L-c \xi$. First, we see a result, assuming also that there exists a solution $u_{m}^{k}$ of (2.1) which satisfies the stability condition called the CFLcondition

$$
\left|H_{p}\left(x_{m}, t_{k}, c+u_{m}^{k}\right)\right|<\lambda^{-1} \quad(\lambda=\Delta t / \Delta x) .
$$

Proposition 2.2. Suppose that we have the solution $v_{m}^{k}$ of (2.3) for which $u_{m}^{k}:=$ $D_{x} v_{m+1}^{k}$ satisfies the CFL-condition for all $m$ and $k=0,1,2, \cdots, k^{*}$. Then the solution is represented for each $n$ and $0<l+1 \leq k^{*}+1$ as

$$
v_{n}^{l+1}=\inf _{\xi} E_{\mu(\cdot ; \xi)}\left[\sum_{0<k \leq l+1} L^{c}\left(\gamma^{k}, t_{k-1}, \xi_{m\left(\gamma^{k}\right)}^{k}\right) \Delta t+v_{\Delta}^{0}\left(\gamma^{0}\right)\right]+h(c) t_{l+1} .
$$

The minimizing velocity field $\xi^{*}$ exists, which is unique and given by

$$
\xi_{m}^{* k+1}=H_{p}\left(x_{m}, t_{k}, c+D_{x} v_{m+1}^{k}\right) .
$$

This proposition is informative, because a proof indicates how the Lax-Friedrichs scheme reveals the stochastic and variational structure. The proof also implies that Proposition 2.2 holds only with the assumptions (A2) and (A3).

Next we remove the assumption of the existence of $v_{m+1}^{k}$ satisfying the CFLcondition:

Theorem 2.3. There exists $\lambda_{1}>0$ (depending on $T,\left[c_{0}, c_{1}\right]$ and $r$, but independent of $\Delta$ ) for which we have the following:

(1) For any small $\Delta=(\Delta x, \Delta t)$ with $\lambda=\Delta t / \Delta x<\lambda_{1}$, the following expectation of the functional with each $n$ and $0<l+1 \leq k(T)$,

$$
E_{n}^{l+1}(\xi):=E_{\mu(\cdot ; \xi)}\left[\sum_{0<k \leq l+1} L^{c}\left(\gamma^{k}, t_{k-1}, \xi_{m\left(\gamma^{k}\right)}^{k}\right) \Delta t+v_{\Delta}^{0}\left(\gamma^{0}\right)\right]+h(c) t_{l+1}
$$

has the infimum denoted by $V_{n}^{l+1}$ with respect to $\xi: G \rightarrow\left[-\lambda^{-1}, \lambda^{-1}\right]$. The infimum $V_{n}^{l+1}$ is attained by $\xi^{*}$ which satisfies $\left|\xi^{*}\right| \leq \lambda_{1}^{-1}<\lambda^{-1}$.

(2) Define $v_{m+1}^{k}$ for each $m$ and $0 \leq k \leq k(T)$ as $v_{m+1}^{0}:=v_{\Delta}^{0}\left(x_{m+1}\right), v_{m+1}^{k}:=$ $V_{m+1}^{k}$. Then the minimizing velocity field $\xi^{*}$ for each $V_{n}^{l+1}$ satisfies in $G$,

$$
L_{\xi}^{c}\left(x_{m}, t_{k}, \xi_{m}^{* k+1}\right)=D_{x} v_{m+1}^{k} \Leftrightarrow \xi_{m}^{* k+1}=H_{p}\left(x_{m}, t_{k}, c+D_{x} v_{m+1}^{k}\right) .
$$

(3) The above $v_{m+1}^{k}$ satisfies (2.3) for $0 \leq k \leq k(T)$.

This theorem immediately leads to one of our main results:

Theorem 2.4. There exists $\lambda_{1}>0$ (depending on $T,\left[c_{0}, c_{1}\right]$ and $r$, but independent of $\Delta$ ) such that for any small $\Delta=(\Delta x, \Delta t)$ with $\lambda=\Delta t / \Delta x<\lambda_{1}$ we have the solution $u_{m}^{k}$ of (2.1) which is bounded and satisfies the CFL-condition up to $k=k(T)$ :

$$
\left|H_{p}\left(x_{m}, t_{k}, c+u_{m}^{k}\right)\right| \leq \lambda_{1}^{-1}<\lambda^{-1} .
$$

Next we "represent" the solution $u_{m}^{k}$ of (2.1): 
Theorem 2.5. For each $n$ and $0<l+1 \leq k(T)$, let $\xi^{*}$ be the minimizer for $V_{n}^{l+1}$ and $\gamma, \mu\left(\cdot ; \xi^{*}\right)$ the minimizing random walk for $V_{n}^{l+1}$. Let $\tilde{\xi}^{*}$ be the minimizer for $V_{n+2}^{l+1}$ and $\tilde{\gamma}, \tilde{\mu}\left(\cdot ; \tilde{\xi}^{*}\right)$ the minimizing random walk for $V_{n+2}^{l+1}$. Then $u_{n+1}^{l+1}$ satisfies

$$
\begin{aligned}
& u_{n+1}^{l+1} \leq E_{\mu\left(\cdot ; \xi^{*}\right)}\left[\sum_{0<k \leq l+1} L_{x}^{c}\left(\gamma^{k}, t_{k-1}, \xi_{m\left(\gamma^{k}\right)}^{* k}\right) \Delta t+u_{\Delta}^{0}\left(\gamma^{0}+\Delta x\right)\right]+O(\Delta x), \\
& u_{n+1}^{l+1} \geq E_{\tilde{\mu}\left(\cdot ; \tilde{\xi}^{*}\right)}\left[\sum_{0<k \leq l+1} L_{x}^{c}\left(\tilde{\gamma}^{k}, t_{k-1}, \tilde{\xi}_{m\left(\tilde{\gamma}^{k}\right)}^{* k}\right) \Delta t+u_{\Delta}^{0}\left(\tilde{\gamma}^{0}-\Delta x\right)\right]+O(\Delta x),
\end{aligned}
$$

where $O(\Delta x)$ stands for a number of $(-\theta \Delta x, \theta \Delta x)$ with $\theta>0$ independent of $\Delta x$.

2.4. Convergence of approximation. We present convergence results based on the dynamical approach to the Lax-Friedrichs scheme. We always take the limit $\Delta=(\Delta x, \Delta t) \rightarrow 0$ under hyperbolic scaling $0<\lambda_{0} \leq \lambda=\Delta t / \Delta x<\lambda_{1}$. We say that a point $(x, t) \in \mathbb{T} \times(0, T]$ is a regular point, if $v_{x}(x, t)$ exists. Note that regular points are nothing but points of continuity of $u=v_{x}$ and almost every point is regular. The minimizing curve of $v(x, t)$ is unique, if $(x, t)$ is regular (see e.g. [3]).

Theorem 2.6. Let $v$ be the viscosity solution of (1.2) and let $v_{\Delta}$ be the linear interpolation of the solution $v_{m+1}^{k}$ of (2.3). Then

$$
v_{\Delta} \rightarrow v \text { uniformly on } \mathbb{T} \times[0, T] \text { as } \Delta \rightarrow 0 .
$$

In particular, we have an error estimate: There exists $\beta>0$ independent of $\Delta$ such that

$$
\left\|v_{\Delta}-v\right\|_{C^{0}(\mathbb{T} \times[0, T])} \leq \beta \sqrt{\Delta x}
$$

This result is consistent with the earlier literature cited in the Introduction. The argument is based on a different viewpoint that the random walks become deterministic and our stochastic calculus of variations tend to the deterministic ones as $\Delta \rightarrow 0$.

Theorem 2.7. Let $(x, t) \in \mathbb{T} \times(0, T]$ be a regular point and $\gamma^{*}:[0, t] \rightarrow \mathbb{R}$ the minimizing curve for $v(x, t)$. Let $\left(x_{n}, t_{l+1}\right)$ be a point of $[x-2 \Delta x, x+2 \Delta x) \times$ $[t-\Delta t, t+\Delta t)$ and $\gamma_{\Delta}:[0, t] \rightarrow \mathbb{R}$ the linear interpolation of the random walk $\gamma$ generated by the minimizing velocity field $\xi^{*}$ for $v_{n}^{l+1}$. Then

$$
\gamma_{\Delta} \rightarrow \gamma^{*} \text { uniformly on }[0, t] \text { in probability as } \Delta \rightarrow 0 \text {. }
$$

In particular, the average of $\gamma_{\Delta}$ converges uniformly to $\gamma^{*}$ as $\Delta \rightarrow 0$.

The minimizing curve $\gamma^{*}$ is the genuine backward characteristic curves of $u, v$ starting from $(x, t)$.

Theorem 2.8. Let $u=v_{x}$ be the entropy solution of (1.1) and $u_{\Delta}$ the step function derived from the solution $u_{m}^{k}$ of (2.1), namely $u_{\Delta}(x, t)=u_{m}^{k}$ for $(x, t) \in\left[x_{m}-\right.$ $\left.\Delta x, x_{m}+\Delta x\right) \times\left[t_{k}, t_{k}+\Delta t\right)$. Then for each regular point $(x, t) \in \mathbb{T} \times[0, T]$,

$$
u_{\Delta}(x, t) \rightarrow u(x, t) \text { as } \Delta \rightarrow 0 .
$$

In particular, $u_{\Delta}$ converges uniformly to $u$ on $(\mathbb{T} \times[0, T]) \backslash \Theta$, where $\Theta$ is a neighborhood of the set of points of singularity of $u$ with an arbitrarily small measure.

This convergence result is stronger than the one derived from the usual $L^{1}$ framework in the following sense: The approximate solution $u_{\Delta}$ converges pointwise a.e. and therefore "uniformly" to the particular representative element of the 
$L^{1}$-valued entropy solution, which is the derivative of the corresponding viscosity solution and is represented as (1.4). The Lax-Friedrichs scheme turns out to approximate not only PDE-solutions but also their characteristic curves at the same time.

\section{Proof of RESUlts}

We prove the propositions and theorems in Section 2.

Proof of Proposition 2.1. It follows from (2.2) and (2.4) that $D_{x} v_{m+1}^{0}=u_{m}^{0}$. Subtracting

$$
D_{t} v_{m}^{k+1}+H\left(x_{m}, t_{k}, D_{x} v_{m+1}^{k}\right)=h(c)
$$

from

$$
D_{t} v_{m+2}^{k+1}+H\left(x_{m+2}, t_{k}, D_{x} v_{m+3}^{k}\right)=h(c)
$$

and dividing it by $2 \Delta x$, we see that $D_{x} v_{m+1}^{k}$ is equal to $u_{m}^{k}$.

Converse argument: We inductively define $\tilde{v}_{m+1}^{k}$ in $\mathcal{G}_{\text {odd }}$ as

$$
\tilde{v}_{m+1}^{k}=\tilde{v}_{m-1}^{k}+u_{m}^{k} \cdot 2 \Delta x, \quad \tilde{v}_{-(m+1)}^{k}=\tilde{v}_{-(m-1)}^{k}+u_{-m}^{k} \cdot(-2 \Delta x)
$$

with $\tilde{v}_{1}^{k}:=u_{0}^{k} \Delta x$ for $k=$ even and $\tilde{v}_{0}^{k}:=0$ for $k=$ odd. Then $\tilde{v}_{m+1}^{k}$ satisfies $D_{x} \tilde{v}_{m+1}^{k}=u_{m}^{k}, \tilde{v}_{m+1 \pm 2 N}^{k}=\tilde{v}_{m+1}^{k}$ and $\tilde{v}_{m+1}^{0}=v_{\Delta}^{0}\left(x_{m+1}\right)-v^{0}(0)$. Using the equation of (2.1) and the equalities

$$
\begin{array}{ll}
D_{t} \tilde{v}_{m}^{k+1}=D_{t} \tilde{v}_{0}^{k+1}+\left\{D_{t} u_{1}^{k+1}+D_{t} u_{3}^{k+1}+\cdots+D_{t} u_{m-1}^{k+1}\right\} 2 \Delta x & \text { for } k=\text { even }, \\
D_{t} \tilde{v}_{m}^{k+1}=D_{t} \tilde{v}_{1}^{k+1}+\left\{D_{t} u_{2}^{k+1}+D_{t} u_{4}^{k+1}+\cdots+D_{t} u_{m-1}^{k+1}\right\} 2 \Delta x & \text { for } k=\text { odd }
\end{array}
$$

(note that $D_{t} \tilde{v}_{0}^{k+1}=0$ ), we obtain

$$
D_{t} \tilde{v}_{m}^{k+1}+H\left(x_{m}, t_{k}, c+D_{x} \tilde{v}_{m+1}^{k}\right)=P^{k},
$$

where for $k=$ even, odd,

$$
P^{k}=D_{t} \tilde{v}_{0}^{k+1}+H\left(x_{0}, t_{k}, c+D_{x} \tilde{v}_{1}^{k}\right), \quad P^{k}=D_{t} \tilde{v}_{1}^{k+1}+H\left(x_{1}, t_{k}, c+D_{x} \tilde{v}_{2}^{k}\right) .
$$

We define $v_{m+1}^{k}$ as

$$
v_{m+1}^{0}:=\tilde{v}_{m+1}^{0}+v^{0}(-\Delta x), \quad v_{m+1}^{k}:=\tilde{v}_{m+1}^{k}-\sum_{0 \leq k^{\prime}<k}\left(P^{k^{\prime}}-h(c)\right) \Delta t .
$$

Then $v_{m+1}^{k}$ satisfies (2.3) and $D_{x} v_{m+1}^{k}=u_{m}^{k}$.

Define $\Omega_{m}^{k}:=\left\{\gamma \in \Omega \mid \gamma^{k}=x_{m}\right\}$ and $p_{m}^{k}:=\sum_{\gamma \in \Omega_{m}^{k}} \mu(\gamma)$. We observe the following lemma:

Lemma 3.1. (1) $\sum_{x \in X^{k}} p_{m(x)}^{k}=1$. Hence $\left\{p_{m(x)}^{k}\right\}_{x \in X^{k}}$ yields a probability measure of $X^{k}$.

(2) $p_{m}^{k}=p_{m-1}^{k+1} \overline{\bar{\rho}}_{m-1}^{k+1}+p_{m+1}^{k+1} \bar{\rho}_{m+1}^{k+1}$, where $\overline{\bar{\rho}}_{m \pm 1}^{k+1}, \bar{\rho}_{m \pm 1}^{k+1}=0$ if $x_{m \pm 1} \notin X^{k+1}$.

This follows from the definition of random walks. 
Proof of Proposition 2.2. Fix $\xi: G \rightarrow\left[-\lambda^{-1}, \lambda^{-1}\right]$ arbitrarily. It follows from the difference equation of (2.3) and the property of the Legendre transform that

$$
\begin{aligned}
v_{n}^{l+1}= & \frac{v_{n-1}^{l}+v_{n+1}^{l}}{2}-H\left(x_{n}, t_{l}, c+D_{x} v_{n+1}^{l}\right) \Delta t+h(c) \Delta t \\
= & \left\{\xi_{n}^{l+1} \cdot\left(c+D_{x} v_{n+1}^{l}\right)-H\left(x_{n}, t_{l}, c+D_{x} v_{n+1}^{l}\right)\right\} \Delta t-c \xi_{n}^{l+1} \Delta t \\
& +\left(\frac{1}{2}+\frac{1}{2} \lambda \xi_{n}^{l+1}\right) v_{n-1}^{l}+\left(\frac{1}{2}-\frac{1}{2} \lambda \xi_{n}^{l+1}\right) v_{n+1}^{l}+h(c) \Delta t \\
\leq & L^{c}\left(x_{n}, t_{l}, \xi_{n}^{l+1}\right) \Delta t+\left(\frac{1}{2}+\frac{1}{2} \lambda \xi_{n}^{l+1}\right) v_{n-1}^{l}+\left(\frac{1}{2}-\frac{1}{2} \lambda \xi_{n}^{l+1}\right) v_{n+1}^{l}+h(c) \Delta t,
\end{aligned}
$$

where the equality holds, if and only if $\xi_{n}^{l+1}=H_{p}\left(x_{n}, t_{l}, c+D_{x} v_{n+1}^{l}\right) \in\left(-\lambda^{-1}, \lambda^{-1}\right)$. Similarly we have

$$
\begin{aligned}
v_{n-1}^{l} \leq & L^{c}\left(x_{n-1}, t_{l-1}, \xi_{n-1}^{l}\right) \Delta t+\left(\frac{1}{2}+\frac{1}{2} \lambda \xi_{n-1}^{l}\right) v_{n-2}^{l-1}+\left(\frac{1}{2}-\frac{1}{2} \lambda \xi_{n-1}^{l}\right) v_{n}^{l-1} \\
& +h(c) \Delta t, \\
v_{n+1}^{l} \leq & L^{c}\left(x_{n+1}, t_{l-1}, \xi_{n+1}^{l}\right) \Delta t+\left(\frac{1}{2}+\frac{1}{2} \lambda \xi_{n+1}^{l}\right) v_{n}^{l-1}+\left(\frac{1}{2}-\frac{1}{2} \lambda \xi_{n+1}^{l}\right) v_{n+2}^{l-1} \\
& +h(c) \Delta t,
\end{aligned}
$$

where the equality holds, if and only if

$$
\xi_{n \pm 1}^{l}=H_{p}\left(x_{n \pm 1}, t_{l-1}, c+D_{x} v_{n \pm 1+1}^{l-1}\right) \in\left(-\lambda^{-1}, \lambda^{-1}\right) .
$$

Hence, by Lemma 3.1, we obtain

$$
\begin{aligned}
v_{n}^{l+1} \leq & \sum_{\substack{l \leq k \leq l+1\\
}}\left(\sum_{x \in X^{k}} p_{m(x)}^{k} L^{c}\left(x, t_{k-1}, \xi_{m(x)}^{k}\right)\right) \Delta t+\sum_{x \in X^{l-1}} p_{m(x)}^{l-1} v_{m(x)}^{l-1} \\
& +h(c)\left(t_{l+1}-t_{l-1}\right) .
\end{aligned}
$$

Continuing this process, we obtain

$$
v_{n}^{l+1} \leq \sum_{0<k \leq l+1}\left(\sum_{x \in X^{k}} p_{m(x)}^{k} L^{c}\left(x, t_{k-1}, \xi_{m(x)}^{k}\right)\right) \Delta t+\sum_{x \in X^{0}} p_{m(x)}^{0} v_{m(x)}^{0}+h(c) t_{l+1} .
$$

The equality holds, if and only if

$$
\xi_{m}^{k}=H_{p}\left(x_{m}, t_{k-1}, c+D_{x} v_{m+1}^{k-1}\right) \in\left(-\lambda^{-1}, \lambda^{-1}\right) .
$$

We see that the first and second term of the right-hand side, denoted by $A_{1}$ and $A_{2}$, become

$$
\begin{aligned}
A_{1} & =\sum_{0<k \leq l+1}\left\{\sum_{x \in X^{k}}\left(\sum_{\gamma \in \Omega_{m(x)}^{k}} \mu(\gamma ; \xi)\right) L^{c}\left(\gamma^{k}, t_{k-1}, \xi_{m\left(\gamma^{k}\right)}^{k}\right)\right\} \Delta t \\
& =\sum_{0<k \leq l+1}\left(\sum_{\gamma \in \Omega} \mu(\gamma ; \xi) L^{c}\left(\gamma^{k}, t_{k-1}, \xi_{m\left(\gamma^{k}\right)}^{k}\right)\right) \Delta t \\
& =\sum_{\gamma \in \Omega} \mu(\gamma ; \xi)\left(\sum_{0<k \leq l+1} L^{c}\left(\gamma^{k}, t_{k-1}, \xi_{m\left(\gamma^{k}\right)}^{k}\right) \Delta t\right), \\
A_{2} & =\sum_{x \in X^{0}}\left(\sum_{\gamma \in \Omega_{m(x)}^{0}} \mu(\gamma ; \xi)\right) v_{m\left(\gamma^{0}\right)}^{0}=\sum_{\gamma \in \Omega} \mu(\gamma ; \xi) v_{m\left(\gamma^{0}\right)}^{0} .
\end{aligned}
$$

Thus $\xi$ is arbitrary and we conclude (2.5). 
For simplicity, we introduce the following notation: $\left.\xi\right|_{\gamma}: k \mapsto \xi_{m\left(\gamma^{k}\right)}^{k}$,

$$
\begin{aligned}
\mathcal{L}(\gamma, \eta) & :=\int_{0}^{t} L^{c}(\gamma(s), s, \eta(s)) d s+v^{0}(\gamma(0)): A C([0, t]) \times L^{1}([0, t]) \rightarrow \mathbb{R}, \\
\mathcal{L}_{\Delta}(\gamma, \eta) & :=\sum_{0<k \leq l+1} L^{c}\left(\gamma^{k}, t_{k-1}, \eta^{k}\right) \Delta t+v_{\Delta}^{0}\left(\gamma^{0}\right): D \times D \rightarrow \mathbb{R},
\end{aligned}
$$

where $D$ is the set of functions: $\{0,1,2, \cdots, l+1\} \rightarrow \mathbb{R}$.

Proof of Theorem 2.3. It follows from (A1)-(A4) that there exists $\alpha_{1}$ for which we have for any $c \in\left[c_{0}, c_{1}\right]$ that

$$
\left|L_{x}^{c}\right| \leq \alpha_{1}\left(\left|L^{c}\right|+1\right)
$$

and that

$$
L_{*}:=\left|\min \left\{0, \inf _{x, t, \xi, c} L^{c}\right\}\right|
$$

is bounded. Due to the lower boundedness of $L^{c}, E_{n}^{l+1}(\xi)$ has the infimum $V_{n}^{l+1}$ with respect to $\xi: G \rightarrow\left[-\lambda^{-1}, \lambda^{-1}\right]$ for any $\Delta$. Note that, if $\xi^{*}$ is a minimizer for $V_{n}^{l+1}$, then for each $\left(x_{m}, t_{k}\right) \in G V_{m}^{k}$ is attained by $\tilde{\xi}^{*}: \tilde{G} \subset G \rightarrow\left[-\lambda^{-1}, \lambda^{-1}\right]$ which is the restriction of $\xi^{*}$ to $\tilde{G}$ whose vertex is $\left(x_{m}, t_{k}\right)$. Introduce

$$
\begin{aligned}
\alpha_{2} & :=r+T \max _{x, t} L(x, t, 0), \quad \alpha_{3}:=\alpha_{1}\left\{\left(1+2 L_{*}\right) T+\alpha_{2}+r\right\}, \\
(3.1) \frac{1}{\lambda_{1}} & :=\max \left\{\max _{x, t}\left|H_{p}\left(x, t, c_{1}+1+r+\alpha_{3}\right)\right|, \max _{x, t}\left|H_{p}\left(x, t, c_{0}-1-r-\alpha_{3}\right)\right|\right\}, \\
\theta & :=T \max _{x, t,|\xi| \leq \lambda_{1}^{-1}} L_{x x}(x, t, \xi) .
\end{aligned}
$$

Now we assume that, for each $n$ and some $l$ such that $0 \leq l<k(T)$, the minimizer $\xi^{*}$ for $V_{n}^{l+1}$ satisfies $\left|\xi^{*}\right| \leq \lambda_{1}^{-1}$. This is true for $l=0$, because of the boundedness $\left|D_{x} v_{m+1}^{0}\right| \leq r$ and Proposition 2.2. We want to prove the following estimate under $\Delta x \theta \leq 1$ :

$$
\left|D_{x} V_{n+2}^{l+1}\right|=\left|\frac{V_{n+2}^{l+1}-V_{n}^{l+1}}{2 \Delta x}\right| \leq 1+r+\alpha_{3} .
$$

Before proving, we observe the result from (3.2): Consider $E_{n+1}^{l+2}(\xi)$ with $\xi$ whose restriction to $k \leq l+1$ coincides with the minimizers for $V_{n}^{l+1}$ and $V_{n+2}^{l+1}$. Then, using the property of the Legendre transform, we have for any $\xi_{n+1}^{l+2}$,

$$
\begin{aligned}
E_{n+1}^{l+2}(\xi)= & L^{c}\left(x_{n+1}, t_{l+1}, \xi_{n+1}^{l+2}\right) \Delta t+\bar{\rho}_{n+1}^{l+2} V_{n}^{l+1}+\bar{\rho}_{n+1}^{l+2} V_{n+2}^{l+1}+h(c) \Delta t \\
\geq & \left\{\xi_{n+1}^{l+2} \cdot\left(c+D_{x} V_{n+2}^{l+1}\right)-H\left(x_{n+1}, t_{l+1}, c+D_{x} V_{n+2}^{l+1}\right)\right\} \Delta t \\
& -c \xi_{n+1}^{l+2} \Delta t+\left(\frac{1}{2}+\frac{\lambda}{2} \xi_{n+1}^{l+2}\right) V_{n}^{l+1}+\left(\frac{1}{2}-\frac{\lambda}{2} \xi_{n+1}^{l+2}\right) V_{n+2}^{l+1}+h(c) \Delta t \\
= & \frac{V_{n}^{l+1}+V_{n+2}^{l+1}}{2}-H\left(x_{n+1}, t_{l+1}, c+D_{x} V_{n+2}^{l+1}\right) \Delta t+h(c) \Delta t,
\end{aligned}
$$

where the equality holds, if and only if $\xi_{n+1}^{l+2}=H_{p}\left(x_{n+1}, t_{l+1}, c+D_{x} V_{n+2}^{l+1}\right)$. Therefore the minimizer $\xi^{*}$ for $V_{n+1}^{l+2}$ exists and satisfies

$$
\xi_{n+1}^{* l+2}=H_{p}\left(x_{n+1}, t_{l+1}, c+D_{x} V_{n+2}^{l+1}\right) .
$$


In particular, we have $\left|\xi_{n+1}^{* l+2}\right| \leq \lambda_{1}^{-1}$ and $\left|\xi^{*}\right| \leq \lambda_{1}^{-1}$ because of (3.2). Thus, by induction, we complete the proof of (1) and (2).

We prove (3.2): Let $\xi^{*}$ be the minimizer for $V_{n}^{l+1}$ and let $\mu, \gamma, \Omega$, etc., be the notation of the random walks starting from $\left(x_{n}, t_{l+1}\right)$. Let $\tilde{\xi}^{*}$ be the minimizer for $V_{n+2}^{l+1}$ and let $\tilde{\mu}, \tilde{\gamma}, \tilde{\Omega}$, etc., be the notation of the random walks starting from $\left(x_{n+2}, t_{l+1}\right)$. We take $\mu(\cdot ; \zeta)$ with $\zeta\left(x_{m}, t_{k}\right):=\tilde{\xi}^{*}\left(x_{m}+2 \Delta x, t_{k}\right)$. Since $V_{n}^{l+1}$ is the infimum, we have

$V_{n}^{l+1} \leq E_{\mu(\cdot ; \zeta)}\left[\mathcal{L}_{\Delta}\left(\gamma,\left.\zeta\right|_{\gamma}\right)\right]+h(c) t_{l+1}, \quad V_{n+2}^{l+1}=E_{\tilde{\mu}\left(\cdot ; \tilde{\xi}^{*}\right)}\left[\mathcal{L}_{\Delta}\left(\tilde{\gamma},\left.\tilde{\xi}^{*}\right|_{\tilde{\gamma}}\right)\right]+h(c) t_{l+1}$

Since $\Omega=\{\tilde{\gamma}-2 \Delta x \mid \tilde{\gamma} \in \tilde{\Omega}\}$ and $\mu(\tilde{\gamma}-2 \Delta x ; \zeta)=\tilde{\mu}\left(\tilde{\gamma} ; \tilde{\xi}^{*}\right)$, we have

$$
\begin{aligned}
V_{n}^{l+1} \leq & E_{\mu(\cdot ; \zeta)}\left[\mathcal{L}_{\Delta}\left(\gamma,\left.\zeta\right|_{\gamma}\right)\right]+h(c) t_{l+1} \\
= & \sum_{\tilde{\gamma} \in \tilde{\Omega}} \tilde{\mu}\left(\tilde{\gamma} ; \tilde{\xi}^{*}\right)\left[\sum_{0<k \leq l+1} L^{c}\left(\tilde{\gamma}^{k}-2 \Delta x, t_{k-1}, \tilde{\xi}_{m\left(\tilde{\gamma}^{k}\right)}^{* k} \Delta t+v_{m\left(\tilde{\gamma}^{0}-2 \Delta x\right)}^{0}\right]\right. \\
& +h(c) t_{l+1} .
\end{aligned}
$$

Therefore we obtain

$$
\begin{aligned}
D_{x} V_{n+2}^{l+1} \geq & \frac{E_{\tilde{\mu}\left(\cdot ; \tilde{\xi}^{*}\right)}\left[\mathcal{L}_{\Delta}\left(\tilde{\gamma}, \tilde{\xi}^{*} \mid \tilde{\gamma}\right)\right]-E_{\mu(\cdot ; \zeta)}\left[\mathcal{L}_{\Delta}\left(\gamma,\left.\zeta\right|_{\gamma}\right)\right]}{2 \Delta x} \\
= & E_{\tilde{\mu}\left(\cdot ; \tilde{\xi}^{*}\right)}\left[\sum _ { 0 < k \leq l + 1 } \frac { 1 } { 2 \Delta x } \left\{L^{c}\left(\tilde{\gamma}^{k}, t_{k-1}, \tilde{\xi}_{m\left(\tilde{\gamma}^{k}\right)}^{* k}\right)\right.\right. \\
& \left.\left.-L^{c}\left(\tilde{\gamma}^{k}-2 \Delta x, t_{k-1}, \tilde{\xi}_{m\left(\tilde{\gamma}^{k}\right)}^{* k}\right)\right\} \Delta t+\frac{\left.v_{m\left(\tilde{\gamma}^{0}\right)}^{0}-v_{m\left(\tilde{\gamma}^{0}-2 \Delta x\right)}^{0}\right]}{2 \Delta x}\right] \\
= & E_{\tilde{\mu}\left(\cdot ; \tilde{\xi}^{*}\right)}\left[\sum_{0<k \leq l+1} L_{x}^{c}\left(\tilde{\gamma}^{k}, t_{k-1}, \tilde{\xi}_{m\left(\tilde{\gamma}^{k}\right)}^{* k}\right) \Delta t+u_{m\left(\tilde{\gamma}^{0}-\Delta x\right)}^{0}\right] \\
& +E_{\tilde{\mu}\left(\cdot ; \tilde{\xi}^{*}\right)}\left[\sum_{0<k \leq l+1} L_{x x}^{c}\left(\tilde{\gamma}^{k}+\delta\left(\tilde{\gamma}^{k}\right), t_{k-1}, \tilde{\xi}_{m\left(\tilde{\gamma}^{k}\right)}^{* k}\right) \Delta t\right] \Delta x,
\end{aligned}
$$

where $\delta\left(\tilde{\gamma}^{k}\right)$ comes from Taylor's formula. Note that the absolute value of the term on the last line is bounded by $\theta \Delta x$ with $\theta$ independent of $\Delta x$. Using (A4) and noting that $\tilde{\xi}^{*}$ is the minimizer, we see that the term on the fourth line of (3.4) denoted by $A$ is bounded below by $-\left(1+r+\alpha_{3}\right)$ :

$$
\begin{aligned}
A & \geq-E_{\tilde{\mu}\left(\cdot ; \tilde{\xi}^{*}\right)}\left[\sum_{0<k \leq l+1} \alpha_{1}\left(1+\left|L^{c}\left(\tilde{\gamma}^{k}, t_{k-1}, \tilde{\xi}_{m\left(\tilde{\gamma}^{k}\right)}^{* k}\right)\right|\right) \Delta t\right]-r-\theta \Delta x \\
& =-E_{\tilde{\mu}\left(\cdot ; \tilde{\xi}^{*}\right)}\left[\sum_{0<k \leq l+1} \alpha_{1}\left(1+\left|L^{c}\left(\tilde{\gamma}^{k}, t_{k-1}, \tilde{\xi}_{m\left(\tilde{\gamma}^{k}\right)}^{* k}\right)+L_{*}-L_{*}\right|\right) \Delta t\right]-r-\theta \Delta x \\
& \geq-E_{\tilde{\mu}\left(\cdot ; \tilde{\xi}^{*}\right)}\left[\sum_{0<k \leq l+1} \alpha_{1}\left(1+L^{c}\left(\tilde{\gamma}^{k}, t_{k-1}, \tilde{\xi}_{m\left(\tilde{\gamma}^{k}\right)}^{* k}\right)+L_{*}\right) \Delta t\right]-\alpha_{1} L_{*} T-r-1 \\
& =-\alpha_{1} E_{\tilde{\mu}\left(\cdot ; \tilde{\xi}^{*}\right)}\left[\mathcal{L}_{\Delta}\left(\tilde{\gamma}, \tilde{\xi}^{*} \mid \tilde{\gamma}\right)\right]+\alpha_{1} E_{\tilde{\mu}\left(\cdot ; \tilde{\xi}^{*}\right)}\left[v_{\Delta}^{0}\left(\tilde{\gamma}^{0}\right)\right]-\alpha_{1} T-2 \alpha_{1} L_{*} T-r-1 \\
& \geq-\alpha_{1} E_{\tilde{\mu}(\cdot ; 0)}\left[\mathcal{L}_{\Delta}(\tilde{\gamma}, 0 \mid \tilde{\gamma})\right]-\alpha_{1} r-\alpha_{1} T-2 \alpha_{1} L_{*} T-r-1 \\
& \geq-\alpha_{1} \alpha_{2}-\alpha_{1} r-\alpha_{1} T-2 \alpha_{1} L_{*} T-r-1 \\
& =-\left(\alpha_{3}+r+1\right) .
\end{aligned}
$$


Similar reasoning by $\tilde{\mu}(\cdot ; \tilde{\zeta})$ with $\tilde{\zeta}\left(x_{m}, t_{k}\right):=\xi^{*}\left(x_{m}-2 \Delta x, t_{k}\right)$ yields the upper bound of $D_{x} V_{n+2}^{l+1}$. $(3)$.

Since (3.3) becomes an equality for the minimizing velocity field, we conclude

Theorem 2.4 follows from Proposition 2.1 and Theorem 2.3. Theorem 2.5 follows from (3.4) and the similar reasoning for the upper bound of $D_{x} V_{n+2}^{l+1}$.

We observe several properties of the function $v: \mathbb{T} \times[0, T] \rightarrow \mathbb{R}$ defined as

$$
v(x, t):=\inf _{\gamma \in A C, \gamma(t)=x} \mathcal{L}\left(\gamma, \gamma^{\prime}\right)+h(c) t, v(\cdot, 0):=v^{0}(\cdot),
$$

which is the viscosity solution of (1.2). There exists at least one minimizing curve for $v(x, t)$, due to Tonelli's theory.

Lemma 3.2. Let $\gamma^{*}:[0, t] \rightarrow \mathbb{R}$ be a minimizing curve for $v(x, t)$.

(1) The following regularity properties hold:

$$
\begin{aligned}
& L_{\xi}^{c}\left(\gamma^{*}(\tau), \tau, \gamma^{* \prime}(\tau)\right) \in \partial_{x}^{-} v\left(\gamma^{*}(\tau), \tau\right) \text { for } 0 \leq \tau<t, \\
& L_{\xi}^{c}\left(\gamma^{*}(\tau), \tau, \gamma^{* \prime}(\tau)\right) \in \partial_{x}^{+} v\left(\gamma^{*}(\tau), \tau\right) \text { for } 0<\tau \leq t,
\end{aligned}
$$

where $\partial_{x}^{-} v\left(\partial_{x}^{+} v\right)$ is the subdifferential (superdifferential). In particular, $v_{x}\left(\gamma^{*}(\tau), \tau\right)$ exists for $0<\tau<t$ and is equal to $L_{\xi}^{c}\left(\gamma^{*}(\tau), \tau, \gamma^{* \prime}(\tau)\right)$.

(2) $\left|\gamma^{* \prime}(\tau)\right| \leq \lambda_{1}^{-1}$ for $0 \leq \tau \leq t$, where $\lambda_{1}$ is given in (3.1).

(3) $v$ is Lipschitz continuous.

(4) If $(x, t)$ is a regular point, then we have for any $0 \leq \tau<t$,

$u(x, t):=v_{x}(x, t)=\int_{0}^{t} L_{x}^{c}\left(\gamma^{*}(s), s, \gamma^{* \prime}(s)\right) d s+L_{\xi}^{c}\left(\gamma^{*}(\tau), \tau, \gamma^{* \prime}(\tau)\right)$.

If $v^{0}$ is semiconcave or $u^{0}$ is rarefaction-free, then we have

$$
L_{\xi}^{c}\left(\gamma^{*}(0), 0, \gamma^{* \prime}(0)\right)=u^{0}\left(\gamma^{*}(0)\right) .
$$

In particular, $v$ is the viscosity solution of (1.2) and $u$ the entropy solution of (1.1).

Proof. (1), (3), and (4) are known, but for the reader's convenience we give a brief proof.

(1) It follows from the minimizing property of $\gamma^{*}$ that we have for any $0 \leq \tau<t$,

$$
v(x, t)=\int_{\tau}^{t} L^{c}\left(\gamma^{*}(s), s, \gamma^{* \prime}(s)\right) d s+v\left(\gamma^{*}(\tau), \tau\right)+h(c)(t-\tau)
$$

and $\left.\gamma^{*}\right|_{[0, \tau]}$ is a minimizer for $v\left(\gamma^{*}(\tau), \tau\right)$. Hence for any $\varepsilon>0$ such that $\tau+\varepsilon \leq t$ we have

$$
v\left(\gamma^{*}(\tau+\varepsilon), \tau+\varepsilon\right)=\int_{\tau}^{\tau+\varepsilon} L^{c}\left(\gamma^{*}(s), s, \gamma^{* \prime}(s)\right) d s+v\left(\gamma^{*}(\tau), \tau\right)+h(c) \varepsilon .
$$

Define $\gamma:[0, \tau+\varepsilon] \rightarrow \mathbb{R}$ as $\gamma(s):=\gamma^{*}(s)+\frac{\tau+\varepsilon-s}{\varepsilon} \delta$ with $\delta \neq 0$ for $s \in[\tau, \tau+\varepsilon]$ and $\gamma(s):=\tilde{\gamma}^{*}(s)$ for $s \in[0, \tau]$, where $\tilde{\gamma}^{*}$ is a minimizing curve for $v\left(\gamma^{*}(\tau)+\delta, \tau\right)$. Then we have

$$
v\left(\gamma^{*}(\tau+\varepsilon), \tau+\varepsilon\right) \leq \int_{\tau}^{\tau+\varepsilon} L^{c}\left(\gamma(s), s, \gamma^{\prime}(s)\right) d s+v\left(\gamma^{*}(\tau)+\delta, \tau\right)+h(c) \varepsilon .
$$


Therefore we obtain

$$
v\left(\gamma^{*}(\tau)+\delta, \tau\right)-v\left(\gamma^{*}(\tau), \tau\right) \geq L_{\xi}^{c}\left(\gamma^{*}(\tau), \tau, \gamma^{* \prime}(\tau)\right) \delta+O(\varepsilon \delta)+O\left(\frac{\delta^{2}}{\varepsilon}\right),
$$

which means that for $\varepsilon=\sqrt{\delta}$,

$$
\liminf _{\delta \rightarrow 0} \frac{v\left(\gamma^{*}(\tau)+\delta, \tau\right)-v\left(\gamma^{*}(\tau), \tau\right)-L_{\xi}^{c}\left(\gamma^{*}(\tau), \tau, \gamma^{* \prime}(\tau)\right) \delta}{|\delta|} \geq 0 .
$$

Similar reasoning with $\gamma:[0, \tau] \rightarrow \mathbb{R}$ defined as $\gamma(s):=\gamma^{*}(s)+\frac{s-(\tau-\varepsilon)}{\varepsilon} \delta$ for $s \in[\tau-\varepsilon, \tau]$ and $\gamma(s):=\gamma^{*}(s)$ for $s \in[0, \tau-\varepsilon]$ leads to the second inclusion.

(2) Since $\gamma^{*}$ is a minimizer, we have for $\gamma(s) \equiv x$,

$$
\int_{0}^{t} L^{c}\left(\gamma^{*}(s), s, \gamma^{* \prime}(s)\right) d s+v_{0}\left(\gamma^{*}(0)\right) \leq \int_{0}^{t} L^{c}(x, s, 0) d s+v_{0}(x) \leq \alpha_{2} .
$$

Since $\gamma^{*}$ satisfies the Euler-Lagrange equation $\frac{d}{d t} L_{\xi}^{c}\left(\gamma(t), t, \gamma^{\prime}(t)\right)=L_{x}^{c}\left(\gamma(t), t, \gamma^{\prime}(t)\right)$, we obtain

$$
\begin{aligned}
\left|L_{\xi}^{c}\left(\gamma^{*}(s), s, \gamma^{* \prime}(s)\right)\right| & =\left|L_{\xi}^{c}\left(\gamma^{*}(0), 0, \gamma^{* \prime}(0)\right)+\int_{0}^{t} L_{x}^{c}\left(\gamma^{*}(s), s, \gamma^{* \prime}(s)\right) d s\right| \\
& \leq r+\int_{0}^{t} \alpha_{1}\left(1+\left|L^{c}\left(\gamma^{*}(s), s, \gamma^{* \prime}(s)\right)\right|\right) d s \\
& \leq \alpha_{3}+r+1 .
\end{aligned}
$$

(3) Follows from (2).

(4) We observe that for any $0<\tau<t$ and $\delta \neq 0$,

$$
\begin{aligned}
v(x+\delta, t)-v(x, t) \leq & \int_{\tau}^{t} L^{c}\left(\gamma^{*}(s)+\delta, s, \gamma^{* \prime}(s)\right)-L^{c}\left(\gamma^{*}(s), s, \gamma^{* \prime}(s)\right) d s \\
& +v\left(\gamma^{*}(\tau)+\delta, \tau\right)-v\left(\gamma^{*}(\tau), \tau\right) .
\end{aligned}
$$

Therefore, using (1), we obtain

$$
u(x, t)=v_{x}(x, t) \leq \int_{\tau}^{t} L_{x}^{c}\left(\gamma^{*}(s), s, \gamma^{* \prime}(s)\right) d s+L_{\xi}^{c}\left(\gamma^{*}(\tau), \tau, \gamma^{* \prime}(\tau)\right),
$$

which holds for $\tau=0$.

For the converse inequality, we take a sequence $\delta_{j} \rightarrow 0$ such that each $\left(x+\delta_{j}, t\right)$ is a regular point of $v$. Let $\gamma_{j}$ be the minimizer of $v\left(x+\delta_{j}, t\right)$. Then we have $\lim _{j \rightarrow \infty} v_{x}\left(x+\delta_{j}, t\right)=v_{x}(x, t)$. Therefore it holds that $\left(\gamma_{j}, \gamma_{j}^{\prime}\right) \rightarrow\left(\gamma, \gamma^{\prime}\right)$ uniformly as $j \rightarrow \infty$. Similar reasoning with $\gamma_{j}$ yields

$$
u(x, t)=v_{x}(x, t) \geq \int_{\tau}^{t} L_{x}^{c}\left(\gamma^{*}(s), s, \gamma^{* \prime}(s)\right) d s+L_{\xi}^{c}\left(\gamma^{*}(\tau), \tau, \gamma^{* \prime}(\tau)\right),
$$

which holds for $\tau=0$. We refer to 3 . for the fact that $v$ is the viscosity solution of (1.2) and $u$ the entropy solution of (1.1).

We state a result of hyperbolic scaling limit of random walks. We introduce a random variable $\eta(\gamma)=\left\{\eta^{k}(\gamma)\right\}_{k=0,1,2, \cdots, l+1}, \gamma \in \Omega$ which is induced by a random walk with a velocity field $\xi$ and is defined as

$$
\eta^{l+1}:=\gamma^{l+1}, \quad \eta^{k}(\gamma):=\gamma^{l+1}-\sum_{k<k^{\prime} \leq l+1} \xi\left(t_{k^{\prime}}, \gamma^{k^{\prime}}\right) \Delta t \quad \text { for } 0 \leq k \leq l .
$$


Lemma $3.3([18])$. Set $\tilde{\sigma}^{k}:=E_{\mu(\cdot ; \xi)}\left[\left|\gamma^{k}-\eta^{k}(\gamma)\right|^{2}\right]$ and $\tilde{d}^{k}:=E_{\mu(\cdot ; \xi)}\left[\left|\gamma^{k}-\eta^{k}(\gamma)\right|\right]$ for $0 \leq k \leq l+1$. Then we have

$$
\left(\tilde{d}^{k}\right)^{2} \leq \tilde{\sigma}^{k} \leq \frac{t_{l+1}-t_{k}}{\lambda} \Delta x
$$

Remark. We always have $\tilde{\sigma}^{k} \rightarrow 0$ as $\Delta \rightarrow 0$ for any $\xi$ under hyperbolic scaling. However, the variance does not always tend to 0 (see [18]). This estimate is analogous to the following: Let $\eta^{\nu}$ be the stochastic process induced by the solution $\gamma^{\nu}$ of (1.8) as $\eta^{\nu \prime}(s)=\xi^{\nu}\left(s, \gamma^{\nu}(s)\right), \eta^{\nu}(t)=x$. Then we have for $s \in[0, t]$,

$$
E\left[\left|\eta^{\nu}(s)-\gamma^{\nu}(s)\right|\right]=\sqrt{2 \nu} E[|B(t-s)|] .
$$

Proof of Theorem 2.6. Since $v_{\Delta}$ and $v$ is Lipschitz continuous, it is enough to show that $\left|v_{n}^{l+1}-v\left(x_{n}, t_{l+1}\right)\right|=O(\sqrt{\Delta x})$ for all $0 \leq l+1 \leq k(T)$ and $n$. Hereafter, $\beta_{1}, \beta_{2}, \cdots$ are constants independent of $\Delta x, \Delta t, n, l$. Let $\gamma^{*}$ be a minimizer for $v\left(x_{n}, t_{l+1}\right)$. By (2) of Lemma 3.2, we have $\left|\gamma^{* \prime}\right| \leq \lambda_{1}^{-1}<\lambda^{-1}$. We take

$$
\xi\left(x_{m}, t_{k}\right):=\gamma^{* \prime}\left(t_{k}\right),
$$

which yields a space-homogeneous random walk. Then we have

$$
v\left(x_{n}, t_{l+1}\right)=\mathcal{L}\left(\gamma^{*}, \gamma^{* \prime}\right)+h(c) t_{l+1}, \quad v_{n}^{l+1} \leq E_{\mu(\cdot ; \xi)}\left[\mathcal{L}_{\Delta}\left(\gamma,\left.\xi\right|_{\gamma}\right)\right]+h(c) t_{l+1}
$$

Since $\xi$ is space-homogeneous, $\eta(\gamma)$ and $\mathcal{L}_{\Delta}\left(\eta(\gamma),\left.\xi\right|_{\gamma}\right)$ are independent of $\gamma$ and satisfy

$$
\begin{aligned}
\left|\gamma^{*}\left(t_{k}\right)-\eta^{k}(\gamma)\right| & \leq \beta_{1} \Delta x \text { for } 0 \leq k \leq l+1 \\
\left|\mathcal{L}\left(\gamma^{*}, \gamma^{* \prime}\right)-\mathcal{L}_{\Delta}\left(\eta(\gamma),\left.\xi\right|_{\gamma}\right)\right| & \leq \beta_{2} \Delta x+\left|v^{0}\left(\gamma^{*}(0)\right)-v_{\Delta}^{0}\left(\eta^{0}(\gamma)\right)\right| \leq \beta_{3} \Delta x .
\end{aligned}
$$

By Lemma 3.3. we obtain

$$
\begin{aligned}
v_{n}^{l+1}- & v\left(x_{n}, t_{l+1}\right) \leq E_{\mu(\cdot ; \xi)}\left[\mathcal{L}_{\Delta}\left(\gamma,\left.\xi\right|_{\gamma}\right)-\mathcal{L}_{\Delta}\left(\eta(\gamma),\left.\xi\right|_{\gamma}\right)\right]+\beta_{3} \Delta x \\
= & E_{\mu(\cdot ; \xi)}\left[\sum_{0<k \leq l+1}\left\{L^{c}\left(\gamma^{k}, t_{k-1}, \gamma^{* \prime}\left(t_{k}\right)\right)-L^{c}\left(\eta^{k}(\gamma), t_{k-1}, \gamma^{* \prime}\left(t_{k}\right)\right)\right\} \Delta t\right. \\
& \left.\quad+v_{\Delta}^{0}\left(\gamma^{0}\right)-v_{\Delta}^{0}\left(\eta^{0}(\gamma)\right)\right]+\beta_{3} \Delta x \\
\leq & E_{\mu(\cdot ; \xi)}\left[\sum_{0<k \leq l+1} \beta_{4}\left|\gamma^{k}-\eta^{k}(\gamma)\right| \Delta t+r\left|\gamma^{0}-\eta^{0}(\gamma)\right|\right]+\beta_{3} \Delta x \\
\leq & \beta_{5} \sqrt{\Delta x} .
\end{aligned}
$$

Let $\xi^{*}$ be the minimizer for $v_{n}^{l+1}$, which yields a space-time inhomogeneous random walk. Let $\eta_{\Delta}(\gamma):\left[0, t_{l+1}\right] \rightarrow \mathbb{R}$ be the linear interpolation of $\eta(\gamma)$. Then we have

$$
\begin{aligned}
& v_{n}^{l+1}=E_{\mu\left(\cdot ; \xi^{*}\right)}\left[\mathcal{L}_{\Delta}\left(\gamma,\left.\xi^{*}\right|_{\gamma}\right)\right]+h(c) t_{l+1}, \\
& v\left(x_{n}, t_{l+1}\right) \leq \mathcal{L}\left(\eta_{\Delta}(\gamma), \eta_{\Delta}(\gamma)^{\prime}\right)+h(c) t_{l+1} \quad \text { for each } \gamma \in \Omega, \\
& \left|\mathcal{L}\left(\eta_{\Delta}(\gamma), \eta_{\Delta}(\gamma)^{\prime}\right)-\mathcal{L}_{\Delta}\left(\eta(\gamma),\left.\xi^{*}\right|_{\gamma}\right)\right| \leq \beta_{6} \Delta x \quad \text { for each } \gamma \in \Omega .
\end{aligned}
$$


By Lemma 3.3, we obtain

$$
\begin{aligned}
v_{n}^{l+1}- & v\left(x_{n}, t_{l+1}\right) \geq E_{\mu\left(\cdot ; \xi^{*}\right)}\left[\mathcal{L}_{\Delta}\left(\gamma,\left.\xi^{*}\right|_{\gamma}\right)-\mathcal{L}_{\Delta}\left(\eta(\gamma),\left.\xi^{*}\right|_{\gamma}\right)\right]-\beta_{6} \Delta x \\
= & E_{\mu\left(\cdot ; \xi^{*}\right)}\left[\sum_{0<k \leq l+1}\left\{L^{c}\left(\gamma^{k}, t_{k-1}, \xi_{m\left(\gamma^{k}\right)}^{* k}\right)-L^{c}\left(\eta^{k}(\gamma), t_{k-1}, \xi_{m\left(\gamma^{k}\right)}^{* k}\right)\right\} t\right. \\
& \left.\quad+v_{\Delta}^{0}\left(\gamma^{0}\right)-v_{\Delta}^{0}\left(\eta^{0}(\gamma)\right)\right]-\beta_{6} \Delta x \\
\geq & -E_{\mu\left(\cdot ; \xi^{*}\right)}\left[\sum_{0<k \leq l+1} \beta_{4}\left|\gamma^{k}-\eta^{k}(\gamma)\right| \Delta t+r\left|\gamma^{0}-\eta^{0}(\gamma)\right|\right]-\beta_{6} \Delta x \\
\geq & -\beta_{7} \sqrt{\Delta x} .
\end{aligned}
$$

In order to prove the convergence of minimizing random walks to minimizing curves, we observe the following lemmas:

Lemma 3.4. Let $\gamma^{*}$ be the unique minimizer for $v(x, t)$. We define for $\varepsilon>0, b>0$,

$$
\begin{array}{r}
\Gamma^{\varepsilon}:=\left\{\gamma:[0, t] \rightarrow \mathbb{R}|\gamma \in \operatorname{Lip},| \gamma(t)-\gamma^{*}(t)|\leq \varepsilon,| \gamma^{\prime}(s) \mid \leq b,\right. \\
\left.\mathcal{L}\left(\gamma, \gamma^{\prime}\right) \leq \mathcal{L}\left(\gamma^{*}, \gamma^{* \prime}\right)+\varepsilon\right\} .
\end{array}
$$

Then we have

$$
\sup _{\gamma \in \Gamma^{\varepsilon}}\left\|\gamma-\gamma^{*}\right\|_{C^{0}([0, t])} \rightarrow 0, \quad \sup _{\gamma \in \Gamma^{\varepsilon}}\left\|\gamma^{\prime}-\gamma^{* \prime}\right\|_{L^{2}([0, t])} \rightarrow 0 \quad \text { as } \varepsilon \rightarrow 0 .
$$

Proof. Suppose that we have a sequence $\varepsilon_{j} \rightarrow 0$ for which there exists $\delta>0$ such that $\sup _{\gamma \in \Gamma^{\varepsilon_{j}}}\left\|\gamma-\gamma^{*}\right\|_{C^{0}} \geq 2 \delta$ for all $j$. We can take a sequence $\gamma_{j} \in \Gamma^{\varepsilon_{j}}$ for which $\left\|\gamma_{j}-\gamma^{*}\right\|_{C^{0}} \geq \delta$ holds for all $j$. We have a subsequence of $\gamma_{j}$, still denoted by $\gamma_{j}$, such that $\gamma_{j} \rightarrow \tilde{\gamma}$ uniformly with $\left\|\tilde{\gamma}-\gamma^{*}\right\|_{C^{0}} \geq \delta, \tilde{\gamma}(t)=\gamma^{*}(t)=x$ and $\mathcal{L}\left(\tilde{\gamma}, \tilde{\gamma}^{\prime}\right)=\mathcal{L}\left(\gamma^{*}, \gamma^{* \prime}\right)$. Therefore $\tilde{\gamma}$ is another minimizer for $v(x, t)$, which is a contradiction.

Set $\delta(\gamma):=\gamma^{*}(t)-\gamma(t)$ for $\gamma \in \Gamma^{\varepsilon}$. Since $\gamma^{*}$ is the minimizer, we already have $\mathcal{L}\left(\gamma^{*}, \gamma^{* \prime}\right) \leq \mathcal{L}\left(\gamma+\delta(\gamma), \gamma^{\prime}\right)$ and $\left|\mathcal{L}\left(\gamma+\delta(\gamma), \gamma^{\prime}\right)-\mathcal{L}\left(\gamma, \gamma^{\prime}\right)\right| \leq \beta_{8} \varepsilon\left(\beta_{8} \geq 1\right)$ for any $\gamma \in \Gamma^{\varepsilon}$. Hence we have $\varepsilon \geq \mathcal{L}\left(\gamma, \gamma^{\prime}\right)-\mathcal{L}\left(\gamma^{*}, \gamma^{* \prime}\right) \geq-\beta_{8} \varepsilon$. Therefore we obtain

$$
\begin{aligned}
\beta_{8} \varepsilon \geq & \left|\mathcal{L}\left(\gamma, \gamma^{\prime}\right)-\mathcal{L}\left(\gamma^{*}, \gamma^{* \prime}\right)\right| \\
= & \mid \int_{0}^{t}\left\{L^{c}\left(\gamma(s), s, \gamma^{\prime}(s)\right)-L^{c}\left(\gamma^{*}(s), s, \gamma^{\prime}(s)\right)\right. \\
& \left.+L^{c}\left(\gamma^{*}(s), s, \gamma^{\prime}(s)\right)-L^{c}\left(\gamma^{*}(s), s, \gamma^{* \prime}(s)\right)\right\} d s+v^{0}(\gamma(0))-v^{0}\left(\gamma^{*}(0)\right) \mid .
\end{aligned}
$$

Here, by Taylor's formula, we have $\theta(s), \tilde{\theta}(s)$ for which the term \{\} becomes

$$
\begin{aligned}
\{\}= & L_{x}^{c}\left(\gamma^{*}(s)+\theta(s), s, \gamma^{\prime}(s)\right)\left(\gamma(s)-\gamma^{*}(s)\right) \\
& +L_{\xi}^{c}\left(\gamma^{*}(s), s, \gamma^{* \prime}(s)\right)\left(\gamma^{\prime}(s)-\gamma^{* \prime}(s)\right) \\
& +\frac{1}{2} L_{\xi \xi}^{c}\left(\gamma^{*}(s), s, \gamma^{* \prime}(s)+\tilde{\theta}(s)\right)\left(\gamma^{\prime}(s)-\gamma^{* \prime}(s)\right)^{2} .
\end{aligned}
$$


Hence we obtain $\left(L_{\xi \xi}^{c}\left(\gamma^{*}(s), s, \gamma^{* \prime}(s)+\tilde{\theta}(s)\right)\right.$ is abbreviated as $\left.L_{\xi \xi}^{c}\right)$

$$
\begin{gathered}
\beta_{8} \varepsilon \geq \quad-\beta_{9}\left\|\gamma-\gamma^{*}\right\|_{C^{0}} \\
+\left|\int_{0}^{t}\left\{L_{\xi}^{c}\left(\gamma^{*}(s), s, \gamma^{* \prime}(s)\right) \frac{d}{d t}\left(\gamma(s)-\gamma^{*}(s)\right)+\frac{1}{2} L_{\xi \xi}^{c} \cdot\left(\gamma^{\prime}(s)-\gamma^{* \prime}(s)\right)^{2}\right\} d s\right| \\
=\left|L_{\xi}^{c}\left(\gamma^{*}(s), s, \gamma^{* \prime}(s)\right)\left(\gamma(s)-\gamma^{*}(s)\right)\right|_{s=0}^{s=t} \\
\quad-\int_{0}^{t} \frac{d}{d t}\left\{L_{\xi}^{c}\left(\gamma^{*}(s), s, \gamma^{* \prime}(s)\right)\right\}\left(\gamma(s)-\gamma^{*}(s)\right) d s \\
\quad+\int_{0}^{t} \frac{1}{2} L_{\xi \xi}^{c} \cdot\left(\gamma^{\prime}(s)-\gamma^{* \prime}(s)\right)^{2} d s \mid-\beta_{9}\left\|\gamma-\gamma^{*}\right\|_{C^{0}},
\end{gathered}
$$

where the terms on the fourth line can be absorbed in $\left\|\gamma-\gamma^{*}\right\|_{C^{0}}$, since $\gamma^{*}$ satisfies the Euler-Lagrange equation $\frac{d}{d t} L_{\xi}^{c}\left(\gamma^{*}(s), s, \gamma^{* \prime}(s)\right)=L_{x}^{c}\left(\gamma^{*}(s), s, \gamma^{* \prime}(s)\right)$. Since $L_{\xi \xi}^{c}(x, t, \xi)$ is bounded away from zero on each compact set, we see that there exist $\beta_{10}>0, \beta_{11}>0$ such that

$$
\beta_{8} \varepsilon \geq \beta_{10}\left\|\gamma^{\prime}-\gamma^{* \prime}\right\|_{L^{2}}^{2}-\beta_{11}\left\|\gamma-\gamma^{*}\right\|_{C^{0}} .
$$

We have already proved that $\left\|\gamma-\gamma^{*}\right\|_{C^{0}} \rightarrow 0$ as $\varepsilon \rightarrow 0$. This finishes the proof.

Lemma 3.5. Let $f:[0, t] \rightarrow \mathbb{R}$ be a Lipschitz function with a Lipschitz constant $\theta$ satisfying $f(t)=0$. Then we have $\|f\|_{C^{0}([0, t])} \leq \theta\|f\|_{L^{2}([0, t])}+\sqrt{\|f\|_{L^{2}([0, t])}}$.

Proof. We suppose that $\|f\|_{C^{0}}>\sqrt{\|f\|_{L^{2}}}$. Otherwise we get the conclusion. For $I:=\left\{s \in[0, t]|| f(s) \mid \geq \sqrt{\|f\|_{L^{2}}}\right\}$, we have $\|f\|_{L^{2}}^{2} \geq \int_{I}|f(s)|^{2} d s \geq\|f\|_{L^{2}}|I|$ and $|I| \leq\|f\|_{L^{2}}$. The set $I$ necessarily contains $s_{*}$ such that $\left|f\left(s_{*}\right)\right|=\|f\|_{C^{0}}$. Since $f(t)=0$, there always exists $s_{0}>s_{*}$ such that $f\left(s_{0}\right)=\sqrt{\|f\|_{L^{2}}}$ and $\left[s_{*}, s_{0}\right] \subset I$. Hence we have $\left|f\left(s_{0}\right)-f\left(s_{*}\right)\right| \leq \theta\left|s_{0}-s_{*}\right| \leq \theta|I|$. Therefore we obtain $\left|f\left(s_{*}\right)\right| \leq \theta|I|+\left|f\left(s_{0}\right)\right|$.

Proof of Theorem 2.7. Consider $\Omega_{\Delta}^{\varepsilon}:=\left\{\gamma \in \Omega \mid\left\|\gamma_{\Delta}-\gamma^{*}\right\|_{C^{0}} \leq \varepsilon\right\}$, where $\gamma_{\Delta}$ is the linear interpolation of $\gamma$ extended to $s \in[0, t]$. We show that, for each arbitrarily fixed $\varepsilon>0$, we have $\operatorname{prob}\left(\Omega_{\Delta}^{\varepsilon}\right) \rightarrow 1$ as $\Delta \rightarrow 0$.

First we prove the following: We introduce

$$
\Gamma_{\Delta}^{\varepsilon}:=\left\{\gamma \in \Omega \mid\left\|\eta_{\Delta}(\gamma)-\gamma^{*}\right\|_{C^{0}} \leq \varepsilon \text { and }\left\|\eta_{\Delta}(\gamma)^{\prime}-\gamma^{* \prime}\right\|_{L^{2}} \leq \varepsilon\right\},
$$

where $\eta_{\Delta}(\gamma)$ is the linear interpolation of $\eta(\gamma)$ extended to $s \in[0, t]$. Then, for each arbitrarily fixed $\varepsilon>0$, we have $\operatorname{prob}\left(\Gamma_{\Delta}^{\varepsilon}\right) \rightarrow 1$ as $\Delta \rightarrow 0$. In fact, we observe that

$$
\begin{aligned}
v_{n}^{l+1} & =E_{\mu\left(\cdot ; \xi^{*}\right)}\left[\mathcal{L}_{\Delta}\left(\eta(\gamma),\left.\xi^{*}\right|_{\gamma}\right)\right]+h(c) t_{l+1}+O(\sqrt{\Delta x}) \\
& =E_{\mu\left(\cdot ; \xi^{*}\right)}\left[\mathcal{L}\left(\eta_{\Delta}(\gamma), \eta_{\Delta}(\gamma)^{\prime}\right)\right]+h(c) t_{l+1}+O(\sqrt{\Delta x}), \\
v_{n}^{l+1}-v(x, t) & =E_{\mu\left(\cdot ; \xi^{*}\right)}\left[\mathcal{L}_{\Delta}\left(\gamma,\left.\xi^{*}\right|_{\gamma}\right)-\mathcal{L}\left(\gamma^{*}, \gamma^{* \prime}\right)\right]+h(c)\left(t_{l+1}-t\right) \\
& =E_{\mu\left(\cdot ; \xi^{*}\right)}\left[\mathcal{L}\left(\eta_{\Delta}(\gamma), \eta_{\Delta}(\gamma)^{\prime}\right)-\mathcal{L}\left(\gamma^{*}, \gamma^{* \prime}\right)\right]+O(\sqrt{\Delta x}) \\
& =O(\sqrt{\Delta x}),
\end{aligned}
$$


where the last equality comes from Theorem 2.6. Since $\gamma^{*}$ is the minimizer, we have for all $\gamma \in \Omega$,

$$
\begin{aligned}
0 & \leq \mathcal{L}\left(\eta_{\Delta}(\gamma)+x-x_{n}, \eta_{\Delta}(\gamma)^{\prime}\right)-\mathcal{L}\left(\gamma^{*}, \gamma^{* \prime}\right) \\
& =\mathcal{L}\left(\eta_{\Delta}(\gamma), \eta_{\Delta}(\gamma)^{\prime}\right)-\mathcal{L}\left(\gamma^{*}, \gamma^{* \prime}\right)+\beta_{12} \Delta x
\end{aligned}
$$

Consider $\Omega^{+}:=\left\{\gamma \in \Omega \mid \mathcal{L}\left(\eta_{\Delta}(\gamma), \eta_{\Delta}(\gamma)^{\prime}\right)-\mathcal{L}\left(\gamma^{*}, \gamma^{* \prime}\right) \geq \Delta x^{\frac{1}{4}}\right\}$. Then we obtain

$$
\begin{aligned}
O(\sqrt{\Delta x}) & =E_{\mu\left(\cdot ; \xi^{*}\right)}\left[\mathcal{L}\left(\eta_{\Delta}(\gamma), \eta_{\Delta}(\gamma)^{\prime}\right)-\mathcal{L}\left(\gamma^{*}, \gamma^{* \prime}\right)\right] \\
& \geq \sum_{\gamma \in \Omega^{+}} \mu\left(\gamma ; \xi^{*}\right) \Delta x^{\frac{1}{4}}-\beta_{12} \Delta x .
\end{aligned}
$$

Hence $\operatorname{prob}\left(\Omega^{+}\right)=O\left(\Delta x^{\frac{1}{4}}\right)$. It follows from Lemma 3.4 that for $\Delta x \ll \varepsilon$,

$$
\Omega \backslash \Omega^{+} \subset \Gamma_{\Delta}^{\varepsilon} .
$$

Therefore we conclude that $1-\operatorname{prob}\left(\Omega^{+}\right) \leq \operatorname{prob}\left(\Gamma_{\Delta}^{\varepsilon}\right) \leq 1$ and $\operatorname{prob}\left(\Gamma_{\Delta}^{\varepsilon}\right) \rightarrow 1$ as $\Delta \rightarrow 0$. Using this fact and Lemma 3.3 , we obtain

$$
\begin{aligned}
& \left(E_{\mu\left(\cdot ; \xi^{*}\right)}\left[\left\|\gamma_{\Delta}-\gamma^{*}\right\|_{L^{2}}\right]\right)^{2} \leq E_{\mu\left(\cdot ; \xi^{*}\right)}\left[\left\|\gamma_{\Delta}-\gamma^{*}\right\|_{L^{2}}^{2}\right] \\
& \quad \leq 2 E_{\mu\left(\cdot ; \xi^{*}\right)}\left[\left\|\gamma_{\Delta}-\eta_{\Delta}(\gamma)\right\|_{L^{2}}^{2}\right]+2 E_{\mu\left(\cdot ; \xi^{*}\right)}\left[\left\|\eta_{\Delta}(\gamma)-\gamma^{*}\right\|_{L^{2}}^{2}\right] \\
& \quad \rightarrow 0 \quad \text { as } \Delta \rightarrow 0 .
\end{aligned}
$$

Hence, for any $\varepsilon^{\prime}>0$ there exists $\delta>0$ such that if $|\Delta|<\delta$ we have

$$
E_{\mu\left(\cdot ; \xi^{*}\right)}\left[\left\|\gamma_{\Delta}-\gamma^{*}\right\|_{L^{2}}\right] \leq \varepsilon^{\prime} .
$$

Define $\Omega^{++}:=\left\{\gamma \in \Omega \mid\left\|\gamma_{\Delta}-\gamma^{*}\right\|_{L^{2}} \geq \sqrt{\varepsilon^{\prime}}\right\}$. Since

$$
\varepsilon^{\prime} \geq E_{\mu\left(\cdot ; \xi^{*}\right)}\left[\left\|\gamma_{\Delta}-\gamma^{*}\right\|_{L^{2}}\right] \geq \operatorname{prob}\left(\Omega^{++}\right) \sqrt{\varepsilon^{\prime}},
$$

we have $\operatorname{prob}\left(\Omega^{++}\right) \leq \sqrt{\varepsilon^{\prime}}$. Take $\varepsilon^{\prime} \leq O\left(\varepsilon^{4}\right)$. By Lemma 3.5 we have $\left\|\gamma_{\Delta}-\gamma^{*}\right\|_{C^{0}} \leq$ $\varepsilon$ for all $\gamma \in \Omega \backslash \Omega^{++}$. Thus we conclude that $\Omega \backslash \Omega^{++} \subset \Omega_{\Delta}^{\varepsilon}, 1-\sqrt{\varepsilon^{\prime}} \leq \operatorname{prob}\left(\Omega_{\Delta}^{\varepsilon}\right) \leq 1$ and $\operatorname{prob}\left(\Omega_{\Delta}^{\varepsilon}\right) \rightarrow 1$ as $\Delta \rightarrow 0$.

Proof of Theorem 2.8. Let $\left(x_{n}, t_{l+1}\right)$ be such that $x \in\left[x_{n}-\Delta x, x_{n}+\Delta x\right), t \in$ $\left[t_{l+1}, t_{l+2}\right)$. It is enough to show that $u_{n+1}^{l+1} \rightarrow u(x, t)$ as $\Delta \rightarrow 0$. We set

$$
\mathcal{L}^{\prime}\left(\gamma ; \gamma^{\prime}\right):=\int_{0}^{t} L_{x}\left(\gamma(s), s, \gamma^{\prime}(s)\right) d s .
$$

First we assume that $v^{0}$ is semiconcave, or $u^{0}$ is rarefaction-free. Let $\xi^{*}$ be the minimizer for $v_{n}^{l+1}$. It follows from Theorem 2.5 that

$$
u_{n+1}^{l+1} \leq E_{\mu\left(\cdot ; \xi^{*}\right)}\left[\mathcal{L}^{\prime}\left(\eta_{\Delta}(\gamma) ; \eta_{\Delta}(\gamma)^{\prime}\right)+u_{\Delta}^{0}\left(\gamma^{0}+\Delta x\right)\right]+O(\sqrt{\Delta x}),
$$

where $\eta_{\Delta}(\gamma)$ is the linear interpolation of $\eta(\gamma)$ extended to $s \in[0, t]$ that is derived from $\xi^{*}$. Therefore, by (4) of Lemma 3.2, we obtain

$$
\begin{aligned}
u_{n+1}^{l+1}-u(x, t) \leq & E_{\mu\left(\cdot ; \xi^{*}\right)}\left[\mathcal{L}^{\prime}\left(\eta_{\Delta}(\gamma) ; \eta_{\Delta}(\gamma)^{\prime}\right)-\mathcal{L}^{\prime}\left(\gamma^{*} ; \gamma^{* \prime}\right)\right. \\
& \left.+u_{\Delta}^{0}\left(\gamma^{0}+\Delta x\right)-u^{0}\left(\gamma^{*}(0)\right)\right]+O(\sqrt{\Delta x}), \\
u_{\Delta}^{0}\left(\gamma^{0}+\Delta x\right)-u^{0}\left(\gamma^{*}(0)\right)= & \frac{1}{2 \Delta x} \int_{\left(\gamma^{0}+\Delta x\right)-\Delta x}^{\left(\gamma^{0}+\Delta x\right)+\Delta x} u^{0}(y)-u^{0}\left(\gamma^{*}(0)\right) d y .
\end{aligned}
$$


Since $\left\|\eta_{\Delta}(\gamma)-\gamma^{*}\right\|_{C^{0}} \rightarrow 0,\left\|\eta_{\Delta}(\gamma)^{\prime}-\gamma^{* \prime}\right\|_{L^{2}} \rightarrow 0,\left\|\gamma_{\Delta}-\gamma^{*}\right\|_{C^{0}} \rightarrow 0$ as $\Delta \rightarrow 0$ in probability and $u^{0}(y) \rightarrow u^{0}\left(\gamma^{*}(0)\right)$ as $y \rightarrow \gamma^{*}(0)$, we obtain

$$
\limsup _{\Delta \rightarrow 0}\left(u_{n+1}^{l+1}-u(x, t)\right) \leq 0 .
$$

Similar reasoning with the converse inequality in Theorem 2.5 yields

$$
\liminf _{\Delta \rightarrow 0}\left(u_{n+1}^{l+1}-u(x, t)\right) \geq 0 .
$$

We remove the assumption that $v^{0}$ is semiconcave, or remove the assumption that $u^{0}$ is rarefaction-free. We do not always have $u^{0}\left(\gamma^{*}(0)\right)$. By (4) of Lemma 3.2 and Theorem 2.5. we see that for any $0 \leq \tau<\min \left\{t_{l+1}, t\right\}$,

$$
\begin{aligned}
u(x, t) & =\int_{\tau}^{t} L_{x}^{c}\left(\gamma^{*}(s), s, \gamma^{* \prime}(s)\right) d s+L_{\xi}^{c}\left(\gamma^{*}(\tau), \tau, \gamma^{* \prime}(\tau)\right), \\
u_{n+1}^{l+1} & \leq E_{\mu\left(\cdot ; \xi^{*}\right)}\left[\sum_{k(\tau)<k \leq l+1} L_{x}^{c}\left(\gamma^{k}, t_{k-1}, \xi_{m\left(\gamma^{k}\right)}^{* k}\right) \Delta t+D_{x} v_{m\left(\gamma^{k(\tau)}\right)+1}^{k(\tau)}\right]+O(\Delta x) \\
& =E_{\mu\left(\cdot ; \xi^{*}\right)}\left[\sum_{k(\tau)<k \leq l+1} L_{x}^{c}\left(\gamma^{k}, t_{k-1}, \xi_{m\left(\gamma^{k}\right)}^{* k}\right) \Delta t\right. \\
& =E_{\mu\left(\cdot ; \xi^{*}\right)}\left[\int_{\tau}^{t} L_{x}^{c}\left(\eta_{\Delta}(\gamma)(s), s, \eta_{\Delta}^{c}(\gamma)^{\prime}(s)\right) d s\right. \\
& \left.+L_{\xi}^{c}\left(\eta_{\Delta}(\gamma)(\tau), \tau, \eta_{\Delta}(\gamma)^{\prime}(\tau)\right)\right]+O(\sqrt{\Delta x}) .
\end{aligned}
$$

We need some investigation of the estimate

$$
E_{\mu\left(\cdot ; \xi^{*}\right)}\left[\left|L_{\xi}^{c}\left(\eta_{\Delta}(\gamma)(\tau), \tau, \eta_{\Delta}(\gamma)^{\prime}(\tau)\right)-L_{\xi}^{c}\left(\gamma^{*}(\tau), \tau, \gamma^{* \prime}(\tau)\right)\right|\right] .
$$

Note that for any fixed $\varepsilon^{\prime}>0$ we have $\operatorname{prob}\left(\Gamma_{\Delta}^{\varepsilon^{\prime}}\right) \rightarrow 1$ as $\Delta \rightarrow 0$. Hence, for any $\varepsilon>0$ there exists $\delta>0$ such that, if $|\Delta|<\delta$, we have $E_{\mu\left(\cdot ; \xi^{*}\right)}\left[\left\|\eta_{\Delta}(\gamma)^{\prime}-\gamma^{* \prime}\right\|_{L^{2}}\right]<\varepsilon / \sqrt{t}$. Since $\left\|\eta_{\Delta}(\gamma)^{\prime}-\gamma^{* \prime}\right\|_{L^{1}} \leq \sqrt{t}\left\|\eta_{\Delta}(\gamma)^{\prime}-\gamma^{* \prime}\right\|_{L^{2}}$, we have

$$
E_{\mu\left(\cdot ; \xi^{*}\right)}\left[\left\|\eta_{\Delta}(\gamma)^{\prime}-\gamma^{* \prime}\right\|_{L^{1}}\right]<\varepsilon .
$$

Suppose that

$$
E_{\mu\left(\cdot ; \xi^{*}\right)}\left[\left|\eta_{\Delta}(\gamma)^{\prime}(s)-\gamma^{* \prime}(s)\right|\right] \geq \varepsilon / t \quad \text { for a.e. } s \in[0, t] .
$$

Then we see that

$$
\varepsilon \leq \int_{0}^{t} E_{\mu\left(\cdot ; \xi^{*}\right)}\left[\left|\eta_{\Delta}(\gamma)^{\prime}(s)-\gamma^{* \prime}(s)\right|\right] d s=E_{\mu\left(\cdot ; \xi^{*}\right)}\left[\left\|\eta_{\Delta}(\gamma)^{\prime}-\gamma^{* \prime}\right\|_{L^{1}}\right]<\varepsilon,
$$

which is a contradiction. Therefore there exists $\tau^{*} \in[0, t]$ such that

$$
E_{\mu\left(\cdot ; \xi^{*}\right)}\left[\left|\eta_{\Delta}(\gamma)^{\prime}\left(\tau^{*}\right)-\gamma^{* \prime}\left(\tau^{*}\right)\right|\right]<\varepsilon / t
$$

We already know that $\left\|\eta_{\Delta}(\gamma)-\gamma^{*}\right\|_{C^{0}} \rightarrow 0$ as $\Delta \rightarrow 0$ in probability. Taking $\tau=\tau^{*}$, we conclude (3.5). Similar reasoning yields (3.6). 


\section{ACKNOWLEDGEMENTS}

The author would like to thank Professors Takaaki Nishida and Wilhelm Stannat, who both have interest in this work and listened to me. The work was supported by the JSPS Japanese-German Graduate Externship.

\section{REFERENCES}

[1] Nalini Anantharaman, Renato Iturriaga, Pablo Padilla, and Héctor Sánchez-Morgado, Physical solutions of the Hamilton-Jacobi equation, Discrete Contin. Dyn. Syst. Ser. B 5 (2005), no. 3, 513-528, DOI 10.3934/dcdsb.2005.5.513. MR.2151719(2006e:35031)

[2] Ugo Bessi, Aubry-Mather theory and Hamilton-Jacobi equations, Comm. Math. Phys. 235 (2003), no. 3, 495-511, DOI 10.1007/s00220-002-0781-5. MR.1974512(2004e:37096)

[3] Piermarco Cannarsa and Carlo Sinestrari, Semiconcave Functions, Hamilton-Jacobi Equations, and Optimal Control, Progress in Nonlinear Differential Equations and their Applications, 58, Birkhäuser Boston Inc., Boston, MA, 2004. MR2041617 (2005e:49001)

[4] M. G. Crandall and P.-L. Lions, Two approximations of solutions of Hamilton-Jacobi equations, Math. Comp. 43 (1984), no. 167, 1-19, DOI 10.2307/2007396. MR744921 (86j:65121)

[5] Michael G. Crandall and Andrew Majda, Monotone difference approximations for scalar conservation laws, Math. Comp. 34 (1980), no. 149, 1-21, DOI 10.2307/2006218. MR551288 (81b:65079)

[6] Weinan E, Aubry-Mather theory and periodic solutions of the forced Burgers equation, Comm. Pure Appl. Math. 52 (1999), no. 7, 811-828, DOI 10.1002/(SICI)10970312(199907)52:7〈811::AID-CPA2〉3.0.CO;2-D. MR1682812(2000b:37068)

[7] Albert Fathi, Théorème KAM faible et théorie de Mather sur les systèmes lagrangiens (French, with English and French summaries), C. R. Acad. Sci. Paris Sér. I Math. 324 (1997), no. 9, 1043-1046, DOI 10.1016/S0764-4442(97)87883-4. MR1451248 (98g:58151)

[8] A. Fathi, Weak KAM Theorem in Lagrangian Dynamics, Cambridge Univ. Pr. (2011).

[9] Wendell H. Fleming, The Cauchy problem for a nonlinear first order partial differential equation, J. Differential Equations 5 (1969), 515-530. MR0235269 (38 \#3579)

[10] Wendell H. Fleming and H. Mete Soner, Controlled Markov Processes and Viscosity Solutions, 2nd ed., Stochastic Modelling and Applied Probability, vol. 25, Springer, New York, 2006. MR2179357 (2006e:93002)

[11] Renato Iturriaga and Héctor Sánchez-Morgado, Limit of the infinite horizon discounted Hamilton-Jacobi equation, Discrete Contin. Dyn. Syst. Ser. B 15 (2011), no. 3, 623-635, DOI 10.3934/dcdsb.2011.15.623. MR2774130(2012c:49070)

[12] H. R. Jauslin, H. O. Kreiss, and J. Moser, On the forced Burgers equation with periodic boundary conditions, Differential equations: La Pietra 1996 (Florence), Proc. Sympos. Pure Math., vol. 65, Amer. Math. Soc., Providence, RI, 1999, pp. 133-153. MR1662751 (99m:35208)

[13] K. H. Karlsen and J. D. Towers, Convergence of the Lax-Friedrichs scheme and stability for conservation laws with a discontinous space-time dependent flux, Chinese Ann. Math. Ser. B 25 (2004), no. 3, 287-318, DOI 10.1142/S0252959904000299. MR2086124 (2005h:65145)

[14] Takaaki Nishida and Kohei Soga, Difference approximation to Aubry-Mather sets of the forced Burgers equation, Nonlinearity 25 (2012), no. 9, 2401-2422, DOI 10.1088/09517715/25/9/2401. MR2967111

[15] O. A. Oleĭnik, Discontinuous solutions of non-linear differential equations, Amer. Math. Soc. Transl. (2) 26 (1963), 95-172. MR0151737 (27 \#1721)

[16] Florin Şabac, The optimal convergence rate of monotone finite difference methods for hyperbolic conservation laws, SIAM J. Numer. Anal. 34 (1997), no. 6, 2306-2318, DOI 10.1137/S003614299529347X. MR.1480382 (98j:65064)

[17] A. N. Sobolevskiur, Periodic solutions of the Hamilton-Jacobi equation with a periodic nonhomogeneity, and the Aubry-Mather theory (Russian, with Russian summary), Mat. Sb. 190 (1999), no. 10, 87-104, DOI 10.1070/SM1999v190n10ABEH000435; English transl., Sb. Math. 190 (1999), no. 9-10, 1487-1504. MR.1740158 (2000k:35047)

[18] Kohei Soga, Space-time continuous limit of random walk with hyperbolic scaling, Nonlinear Analysis 102 (2014), 264-271. MR.3182814

[19] Kohei Soga, More on stochastic and variational approach to the Lax-Friedrichs scheme (submitted) arXiv:1210.2178. 
[20] Panagiotis E. Souganidis, Approximation schemes for viscosity solutions of Hamilton-Jacobi equations, J. Differential Equations 59 (1985), no. 1, 1-43, DOI 10.1016/0022-0396(85)901366. MR $803085(86 \mathrm{k}: 35028)$

[21] Eitan Tadmor, The large-time behavior of the scalar, genuinely nonlinear Lax-Friedrichs scheme, Math. Comp. 43 (1984), no. 168, 353-368, DOI 10.2307/2008281. MR758188 (86g:65162)

Department of Pure and Applied Mathematics, Waseda University, Tokyo 169-8555, JAPAN

Current address: CNRS-ENS Lyon, UMPA UMR 5669, 69364 Lyon cedex 7, France

E-mail address: kohei.soga@ens-lyon.fr 